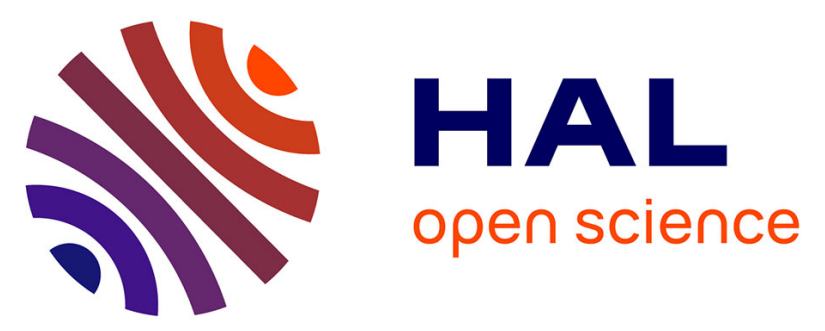

\title{
The root-knot nematode effector MiEFF18 interacts with the plant core spliceosomal protein SmD1 required for giant cell formation
}

\author{
Joffrey Mejias, Jérémie Bazin, Nhat-my Truong, Yongpan Chen, Nathalie \\ Marteu, Nathalie Bouteiller, Shinichiro Sawa, Martin Crespi, Hervé \\ Vaucheret, Pierre Abad, et al.
}

\section{To cite this version:}

Joffrey Mejias, Jérémie Bazin, Nhat-my Truong, Yongpan Chen, Nathalie Marteu, et al.. The rootknot nematode effector MiEFF18 interacts with the plant core spliceosomal protein SmD1 required for giant cell formation. New Phytologist, 2021, 229 (6), pp.3408-3423. 10.1111/nph.17089 . hal03148749

\author{
HAL Id: hal-03148749 \\ https://hal.inrae.fr/hal-03148749
}

Submitted on 8 Dec 2021

HAL is a multi-disciplinary open access archive for the deposit and dissemination of scientific research documents, whether they are published or not. The documents may come from teaching and research institutions in France or abroad, or from public or private research centers.
L'archive ouverte pluridisciplinaire HAL, est destinée au dépôt et à la diffusion de documents scientifiques de niveau recherche, publiés ou non, émanant des établissements d'enseignement et de recherche français ou étrangers, des laboratoires publics ou privés. 
3

The Root-Knot Nematode Effector MiEFF18 interacts with the Plant Core Spliceosomal Protein SmD1 Required for Giant Cell Formation

\section{Joffrey Mejias ${ }^{1}$, Jérémie Bazin ${ }^{2}$, Nhat-My Truong, ${ }^{1,3}$, Yongpan Chen ${ }^{1,4}$, Nathalie} Marteu $^{1}$, Nathalie Bouteiller ${ }^{5}$, Shinichiro Sawa ${ }^{3}$, Martin D. Crespi ${ }^{2}$, Hervé Vaucheret $^{5}$, Pierre Abad ${ }^{1}$, Bruno Favery ${ }^{1, *}$ and Michaël Quentin ${ }^{1, *}$

* co-corresponding authors

${ }^{1}$ INRAE, Université Côte d'Azur, CNRS, ISA, F-06903 Sophia Antipolis, France

${ }^{2}$ Institute of Plant Sciences Paris-Saclay (IPS2), CNRS, INRA, Universités Paris Saclay,-, Evry, Université de Paris, 91192 Gif sur Yvette, France

${ }^{3}$ Graduate School of Science and Technology, Kumamoto University, Kumamoto 860-11 8555, Japan

${ }^{4}$ Department of Plant Pathology and Key Laboratory of Pest Monitoring and Green Management of the Ministry of Agriculture, China Agricultural University, Beijing, China 5 Institut Jean-Pierre Bourgin, INRAE, AgroParisTech, Université Paris-Saclay, 78000 Versailles, France

\section{* Authors for correspondence}

Dr. Michaël Quentin

400 route des chappes, BP 167, 0690 Sophia Antipolis, France

Tel: +33492386495

Email: michael.quentin@inrae.fr

Dr. Bruno Favery

400 route des chappes, BP 167, 0690 Sophia Antipolis, France

Tel: +33492386464

Email: bruno.favery@inrae.fr

Joffrey Mejias, joffrey.mejias@etu.univ-cotedazur.fr, https://orcid.org/0000-0001-7663-0314 Jérémie Bazin, jeremie.bazin@universite-paris-saclay.fr

Nhat-My Truong, truongnhatmy@gmail.com,https://orcid.org/0000-0003-2436-7897 
34 Yongpan Chen, chenyongpan1@163.com, https://orcid.org/0000-0001-9074-7199

$35 \quad$ Nathalie Marteu, nathalie.marteu@inrae.fr

$36 \quad$ Nathalie Bouteiller, nathalie.bouteiller@inrae.fr

37 Shinichiro Sawa, sawa@,kumamoto-u.ac.jp, https://orcid.org/0000-0002-9309-9104

38 Martin D. Crespi, martin.crespi@universite-paris-saclay.fr, https://orcid.org/0000-0002-5698-

$39 \quad 9482$

40 Hervé Vaucheret, herve.vaucheret@inrae.fr, https://orcid.org/0000-0002-9986-0988

$41 \quad$ Pierre Abad pierre.abad@inrae.fr, https://orcid.org/0000-0003-0062-3876

42 Bruno Favery bruno.favery@,inrae.fr, https://orcid.org/0000-0003-3323-1852

43 Michael Quentin michael.quentin@,inrae.fr, https://orcid.org/0000-0002-8030-1203

44

45

\section{Total word count}

46 6,853 words (Introduction, 897; Materials and Methods, 1823; Results, 2143; Discussion, 47 1775; Acknowledgements, 234). Figures: 5 (All figures in colour).

Supporting information files: 23 (12 tables and 11 figures). 


\section{Summary}

- The root-knot nematode Meloidogyne incognita secretes specific effectors (MiEFF) and induces the redifferentiation of plant root cells into enlarged multinucleate feeding "giant cells" essential for nematode development.

- Immunolocalisations revealed the presence of the MiEFF18 protein in the salivary glands of M. incognita juveniles. In planta, MiEFF18 localizes to the nuclei of giant cells demonstrating its secretion during plant-nematode interactions. A yeast two-hybrid approach identified the nuclear ribonucleoprotein SmD1 as a MiEFF18 partner in tomato and Arabidopsis. SmD1 is an essential component of the spliceosome, a complex involved in pre-mRNA splicing and alternative splicing.

- RNA-seq analyses of Arabidopsis roots ectopically expressing MiEFF18 or partially impaired in SmD1 function (smd1b mutant) revealed the contribution of the effector and its target to alternative splicing and proteome diversity. The comparison with Arabidopsis galls data showed that MiEFF18 modifies the expression of genes important for giant cells ontogenesis, indicating that MiEFF18 modulates $\mathrm{SmD1}$ functions to facilitate giant cell formation.

- Finally, Arabidopsis smdlb mutants exhibited less susceptibility to M. incognita infection, and the giant cells formed on these mutants displayed developmental defects, suggesting that SmD1 plays an important role in the formation of giant cells and is required for successful nematode infection.

Key words: Meloidogyne incognita, Effector, Nucleus, Alternative Splicing, Arabidopsis thaliana, Nicotiana benthamiana 


\section{Introduction}

Pathogens have evolved an arsenal of molecules known as effectors, which are secreted in planta to manipulate host functions and ensure successful infection. One striking example of plant cell manipulation is provided by the plant-parasitic root-knot nematodes (RKN) of the genus Meloidogyne. After penetrating the root and migrating to the vascular cylinder, the microscopic vermiform second-stage juveniles $(\mathrm{J} 2 \mathrm{~s})$ induce the transformation of selected vascular root cells into specialised hypertrophied and multinucleate feeding cells. These 'giant cells' result from successive nuclear divisions without cell division, followed by isotropic cell growth (Favery et al., 2016). They are several hundred times larger than normal root cells, contain about 50 to 100 endoreduplicated nuclei and have an expanded endoplasmic reticulum and numerous organelles (de Almeida Engler \& Gheysen, 2013). Giant cells are surrounded by dividing cells, some of which differentiate into new xylem and phloem cells (Bartlem et al., 2014), leading to the formation of a new organ, the gall. Giant cells act as a strong metabolic sink and are the sole source of nutrients for the nematode during the sedentary part of its life cycle in the plant. The pear-shaped RKN females eventually lay their eggs on the root surface. Interestingly, RKN can induce giant cells in more than 4,000 plant species, probably by manipulating conserved plant functions (Singh et al., 2013).

The major modifications observed in giant cells require extensive transcriptional reprogramming in root cells. Giant cell formation has been explored at the transcriptomic level in several plant-RKN interactions, and thousands of differentially expressed genes (DEG) have been identified in plants (Cabrera et al., 2014; Yamaguchi et al., 2017; Shukla et al., 2018; Postnikova et al., 2015; Favery et al., 2016). Functional analyses of these genes have highlighted the key roles of microtubule and actin cytoskeleton rearrangements and cell cycle control in the formation of these multinucleate feeding cells (de Almeida Engler \& Favery, 2011; de Almeida Engler \& Gheysen, 2013; Favery et al., 2016; Cabral et al., 2020). Recent studies have shown that small non-coding RNAs, such as microRNAs (miRNAs) and small interfering RNAs (siRNAs), play a role in gall development (Jaubert-Possamai et al., 2019).

The molecular mechanisms underlying giant cell formation remain poorly understood. It is assumed that effectors, including, in particular, proteins secreted in planta from the three oesophageal gland cells through a hollow protrusive stylet, are responsible for giant cell ontogenesis (Mitchum et al., 2013; Truong et al., 2015; Mejias et al., 2019). Various 
approaches, based on proteomics, transcriptomics and genomics, have been used to characterise RKN effector repertoires. The sequencing of $M$. incognita mRNAs isolated from gland cells or from parasitic juveniles in planta led to the identification of genes encoding putative effector proteins expressed specifically in the oesophageal gland cells and more strongly in planta, such as the Minc18636/Minc15401 genes (Rutter et al., 2014; Nguyen et $a l ., 2018)$. The expression in the oesophageal glands of about a hundred RKN effectors has been validated by in situ hybridisation (ISH), but secretion in planta has been demonstrated for only a few candidate effectors, by immunolocalisation (Truong et al., 2015). Three RKN effectors have been shown to accumulate in the nucleus of giant cells (Jaouannet et al., 2012; Lin et al., 2012; Chen et al., 2017). This targeting event in the host nucleus reflects the need for various nuclear processes, including transcriptional regulation, to be manipulated, to divert plant cell fate and disrupt immunity, as reported for other plant pathogens (Deslandes \& Rivas, 2011; Motion et al., 2015). Most RKN candidate effectors are 'pioneers' displaying no significant sequence similarity to any protein in databases and with no known functional domains; as a result, the functions of only a few RKN effectors have been deciphered (Mejias et al., 2019). The M. incognita 7H08 effector has been shown to have transcriptional activity in planta, but the target genes in the host have yet to be identified (Zhang et al., 2014). The 16D10 effector from M. incognita targets Scarecrow-like transcription factors (Huang et al., 2006). In sedentary endoparasitic cyst nematodes, the GLAND4 effector has been shown to have transcriptional repressor activity against the promoters of two lipid transfer genes involved in plant defence (Barnes et al., 2018). The 32E03 effector has epigenetic activity, through the inhibition of Arabidopsis thaliana histone deacetylases, thereby modulating host rDNA gene expression and promoting infection (Vijayapalani et al., 2018).

Alternative splicing (AS) is a mechanism by which different forms of mature messenger RNA (mRNA) are generated from the same gene, from specific transcripts or through the deletion or retention of an exon/intron sequence (Wilkinson \& Charenton, 2020). This regulatory mechanism results in the production of several related proteins, or isoforms, thereby increasing proteomic diversity. Plant pathogens have been shown to modulate AS (Rigo et al., 2019). We show here that the MiEFF18 effector from M. incognita accumulates in the plant cell nucleus and interacts with an essential component of the spliceosome machinery, the small ribonucleoprotein particle SmD1, in tomato and Arabidopsis. Using a genome-wide transcriptome analysis, we found that MiEFF18 modulated AS, and gene expression, through a partial impairement of SmD1 activity. We also found that related 
alternative splicing events occur in Arabidopsis upon nematode parasitism. Our findings further demonstrate that $\mathrm{SmD1}$ is required for RKN infection and giant cell formation. Thus, MiEFF18 may contribute to giant cell development by modulating the function of a key component of the spliceosome to promote nematode infection.

\section{Materials and methods}

\section{Plant material and growth conditions}

All the $A$. thaliana plants used here were of the Columbia 0 ecotype (Col-0). The smdla and smd $1 b$ mutants have been described elsewhere (Elvira-Matelot et al., 2016). Seeds of $A$. thaliana Col-0, mutant and transgenic lines were surface-sterilised and sown on Murashige and Skoog (Duchefa) agar plates (0.5 x MS salts, $1 \%$ sucrose, $0.8 \%$ agar, $\mathrm{pH} 6.4)$ or in a mixture of soil and sand. Sowings were incubated at $4{ }^{\circ} \mathrm{C}$ for two days, and then transferred to a growth chamber with an $8 \mathrm{~h}$ photoperiod, at $21^{\circ} \mathrm{C}$. For propagation and transformation, seedlings were transferred to a growth chamber with a $16 \mathrm{~h}$ photoperiod, at $21^{\circ} \mathrm{C}$. A. thaliana were transformed by the floral dip method (Bent \& Clough, 1998). Homozygous transformed T3 plants were used. Nicotiana benthamiana plants were grown on soil, under a $16 \mathrm{~h}$ photoperiod, at $24^{\circ} \mathrm{C}$. For the production of plant material for RNA-seq experiments, seeds were surface-sterilized and sown in liquid MS medium (0.5 x MS salts, 1\% sucrose, pH 6.4) with gentle shaking $(70 \mathrm{rpm})$, under a $12 \mathrm{~h}$ photoperiod, at $25^{\circ} \mathrm{C}$. Roots were collected after 11 days and immediately frozen in liquid nitrogen until RNA extraction.

\section{RKN infection assay}

M. incognita strain "Morelos" was multiplied on tomato (Solanum lycopersicum cv. "Saint Pierre") growing in a growth chamber $\left(25^{\circ} \mathrm{C}, 16 \mathrm{~h}\right.$ photoperiod). Freshly hatched $\mathrm{J} 2 \mathrm{~s}$ were collected as previously described (Caillaud \& Favery, 2016). Three-week-old Arabidopsis seedlings were inoculated with 200 M. incognita J2s per plant. Roots were collected six weeks after infection and stained with $0.5 \%$ eosin. The number of females forming egg masses and root weight were then determined ( $\mathrm{n}=25$ to 40 plants per replicates). Three independent biological replicates were established for each set of conditions. Statistical analyses were carried out with $\mathrm{R}$ software ( $\mathrm{R}$ Development Core Team, version 3.1.3). The effect of plant genotype on the number of nematode egg masses was analyzed with generalised linear models (GLMs) based on a Poisson distribution, for each replicate. We 
used the Tukey adjustment method ('multcomp' package) for multiple testing. For giant cell area measurements, galls were collected 14 days post-infection (dpi), cleared in benzyl alcohol/benzyl benzoate (BABB) as previously described (Cabrera et al., 2018) and examined under an inverted confocal microscope (model LSM 880; Zeiss). The mean areas of giant cells in each gall, for each genotype, and for two biological replicates, were measured with Zeiss ZEN software ( $n=42$ and 25 galls for Col-0 and $s m d 1 b$, respectively). The impact of the plant genotype on the giant cell surface was analyzed using student $t$ test since the dependent variables followed a Normal distribution using a Shapiro-Wilk Test.

\section{Plasmid constructs}

The M. incognita MiEFF18 and MiEFF16 coding sequences (CDS) lacking the signal peptide, the S. lycopersicum SmD1, A. thaliana SmD1a and SmD1b, the SV40 Antigen T, and the human P53 sequence were amplified by PCR with specific primers (Table S1) and inserted into the pDON207 donor vector. They were recombined in pK2GW7 (P35S:MiEFF18), pK7WGR2 (P35S:mRFP-MiEFF18), pK7FGW2 (P35S:eGFP-S1SmD1, P35S:eGFP-MiEFF16) or BiFC (pAM-35SS:GWY-YFPc, pAM-35SS:GWY-YFPn, pAM35SS:YFPc-GWY, pAM-35SS:YFPn-GWY) or, for Y2H, the pB27-GW and pP6-GW (Karimi et al., 2007; Caillaud et al., 2009), with Gateway technology (Invitrogen). All the constructs were sequenced (GATC Biotech) and transferred into either Agrobacterium tumefaciens strain GV3101 or Saccharomyces cerevisiae strain L40 AGal4 or Y187.

\section{N. benthamiana agroinfiltration}

Transient expression was achieved by infiltrating $N$. benthamiana leaves with $A$. tumefaciens GV3101 strains harbouring GFP- or mRFP-fusion or BiFC constructs, as previously described (Caillaud et al., 2009). Leaves were imaged 48 hours after agroinfiltration, with an inverted confocal microscope equipped with an Argon ion and HeNe laser as the excitation source. For simultaneous GFP/mRFP imaging, samples were excited at $488 \mathrm{~nm}$ for GFP and $543 \mathrm{~nm}$ for mRFP, in the multi-track scanning mode. GFP or YFP emission was detected selectively with a 505-530 nm band-pass emission filter. We detected mRFP fluorescence in a separate detection channel, with a 560-615 nm band-pass emission filter.

\section{Sequence analysis and alignment}


M. incognita sequences were obtained from Meloidogyne genomic resources (http://www6.inra.fr/meloidogyne_incognita/). We used the MAFFT on the EBI server (https://www.ebi.ac.uk/Tools/msa/mafft/) for sequence alignment. The protein sequences encoded by the genes were analysed with PHOBIUS (http://phobius.sbc.su.se/), PSORT II (http://psort.hgc.jp/form2.html) and NoD (http://www.compbio.dundee.ac.uk/www-nod/) software, for the prediction of signal peptides, non-transmembrane domains, DNA-binding domains, NLS and NoLS, respectively. BLASTp analyses were carried out with an e-value threshold of 0.01 and without low complexity against the NCBI non-redundant protein database, for homologue identification. Interproscan was performed on the proteins to identify protein signatures referenced in the InterPro database (Mitchell et al., 2015).

\section{Yeast two hybrid}

For the yeast two-hybrid (Y2H) screens, the coding sequences of the MiEFF18 and MiEFF16 effectors without their secretion signals and the SlSmD1 CDS were inserted into pB27 as Cterminal fusions with LexA. The constructs were verified by sequencing and used to transform the L40 $\Delta$ Gal4 (MATa) yeast strain. MiEFF18 was used as a bait in a mating approach, to screen a random-primed cDNA library from tomato roots infected with $M$. incognita and Ralstonia solanacearum carried by the Y187 (MAT $\alpha$ ) yeast strain (Hybrigenics Services, Paris, France). Diploids carrying interactions were selected on a minimal synthetic defined SD medium lacking tryptophan (W), leucine (L) and histidine (H). The prey fragments of the positive clones were amplified by PCR and their $5^{\prime}$ junctions were sequenced. The resulting sequences were used to identify the tomato interacting proteins with the Sol Genomics Network (https://solgenomics.net/) blast analysis tools. For pairwise Y2H assays, full-length controls, baits and candidate targets (MiEFF18 w/o SP, MiEFF16 w/o SP, S1SmD1, AtSmD1a, AtSmD1b, Antigen T and P53) were inserted into the pB27 or pP6 vector as C-terminal fusions with LexA or Gal4-AD, respectively, verified by sequencing and used to transform L40 $\Delta$ Gal4 (MATa) or Y187 (MAT $\alpha$ ) yeast strain. After mating between Y187 and L40 $\Delta$ Gal4, diploids were selected on medium lacking tryptophan and leucine, and interactions were tested on medium lacking tryptophan, leucine and histidine and supplemented with $0.5 \mathrm{mM}$ 3-amino-1,2,4-triazole (3-AT).

\section{Western blotting and immunolocalisation}


MiEFF18 was inserted into the pET-24a $(+)$ expression vector (Addgene), expressed in BL21star (DE3) cells, and purified on HisTrap FF columns (GE Healthcare Life Science). The purified protein was used to raise polyclonal antibodies in rabbits (Agro-Bio, La Ferté Saint Aubin, France). Western blotting was performed to check the specificity of the antibody as previously described (Zhao et al., 2019b). Proteins were transferred onto a nitrocellulose membrane with the Trans-Blot Turbo Transfer system (Biorad). The membranes were blocked and incubated with $\alpha$-MiEFF18 antibody $(1: 5,000$ or $1 / 10,000)$ and then with goat anti-rabbit secondary antibodies coupled to horseradish peroxidase (HRP; 1:10,000).

Immunolocalisation was performed directly on M. incognita pre-parasitic J2s with the antiMiEFF18 primary antibody (1:50) and a goat anti-rabbit Alexa Fluor 488-conjugated secondary antibody (1:200) (Molecular Probes) as previously described (Jaubert et al., 2005). Pre-immune serum was used as a negative control. For in planta immunolocalisation, the antibodies were affinity-purified (Agro-Bio, La Ferté Saint Aubin, France) and used to performed immunolocalisation on Arabidopsis gall sections (14 dpi) with the anti-MiEFF18 purified antibody (1:500) and a goat anti-rabbit Alexa Fluor 488-conjugated secondary antibody (1:200) (Molecular Probes) as previously described (Zhao et al., 2019). Images were collected with an inverted confocal microscope (model LSM 880; Zeiss).

\section{Gene expression and alternative splicing analysis}

Arabidopsis gall and non-infected control root RNA-seq data were generated and described in a previous study (Yamaguchi et al., 2017). Total RNA was extracted from the roots of the three Arabidopsis lines (Col-0, P35S:MiEFF18 and smd1b) with TriZol (Invitrogen), according to the Invitrogen protocol. The RNA was treated with DNAse treatment (Ambion), and its quality and integrity were assessed with a Bioanalyzer (Agilent). Libraries were constructed with the Tru-Seq Stranded mRNA Sample Prep kit (Illumina®). Paired-end sequencing with 75-bp reads was performed on a NextSeq500 perform. A minimum of 30 million paired-end reads per sample was generated. RNA-seq preprocessing included the trimming of library adapters and quality controls with Trimmomatic. Paired-end reads with a Phred Quality Score Qscore $>20$ and a read length $>30$ bases were retained, and ribosomal RNA sequences were removed with SortMeRNA. Processed reads were aligned using Tophat2 with the following arguments: --max-multihits 1 -i 20 --min-segment-intron 20 -min-coverage-intron 20 --library-type fr-firststrand --microexon-search -I 1000 --maxsegment-intron 1000 --max-coverage-intron 1000 --b2-very-sensitive. Reads overlapping 
274

275

276

277

278

279

280

281

282

283

284

285

286

287

288

289

290

291

292

293

294

295

296

297

298

299

300

301

302

303

304 305

exons were counted per gene with the FeatureCounts function of the Rsubreads package, using the GTF annotation files from the Araport11 repository (https://www.araport.org/downloads/Araport11_Release_201606/

annotation/Araport11_GFF3_genes_transposons.201606.gff.gz). The significance of differential gene expression was estimated with DEseq2, with FDR correction of the $p$-value during pairwise comparisons between genotypes. A gene was considered to be differentially expressed if its adjusted $p$-value (FDR) was $\leq 0.01$. Transcripts were quantified on the basis of pseudo-alignment counts with kallisto on AtRTD2 transcript sequences (https://ics.hutton.ac.uk/atRTD/RTD2/AtRTDv2_QUASI_19April2016.fa) with a $K$-mer size of 31 nucleotides. Differential AS events in the AtRTD2 database were detected with SUPPA2, using default parameters (Trincado et al., 2018). Only events with an adjusted $p$-val $<0.01$ were retained for further analysis. The dPSI (difference in percent spliced in) values for each AS were generated by SUPPA2 and plotted in R using ggplot2. Hypergeometric pvalue was calculated using the phyper function in R taking the total number AS event as the population size. Gene ontology enrichment analysis was done using the AgriGO server (http://bioinfo.cau.edu.cn/agriGO/) using default parameters. Lists of GO terms were eventually visualized using REVIGO (http://revigo.irb.hr/). Gene family enrichment analysis was performed using GenFam (http://mandadilab.webfactional.com).

\section{Reverse transcription-quantitative PCR}

Total RNA was extracted from plantlets or roots extracted with TriZol (Invitrogen) and subjected ( $1 \mu \mathrm{g}$ of total RNA) to reverse transcription with the Superscript IV reverse transcriptase (Invitrogen). qPCR analyses were performed as described by Nguyen et al. (2018). We performed qPCR on triplicate samples of each cDNA from three independent biological replicates. OXA1 (At5g62050) and UBQ10 (At4g05320) were used for the normalization of RT-qPCR data. Quantifications and statistical analyses were performed with SATqPCR (Rancurel et al., 2019), and the results are expressed as normalised relative quantities. For the validation of alternatively spliced genes, two pairs of primers, specifically amplifying one or the two isoforms of the gene concerned, were designed (Table S1) and used for RT-qPCR assays with the parameters described above for the DEG. The UBQ10 reference gene was used for normalization of the alternatively spliced genes. 


\section{Accession numbers}

Sequence data from this article can be found in the Arabidopsis Information Resource (https://www.arabidopsis.org/), Solgenomics (https://solgenomics.net/) and GenBank/EMBL databases under the following accession numbers: Minc18636 (KX907770), Minc15401 (MT591034), Minc16401 (MT591035), AtSmDla (At3g07590), AtSmD1b (At4g02840), OXA1 (At5g62050), UBQ10 (At4g05320), FAD/NAD(P)-binding oxidoreductase (At5g11330), U1 snRNP 70K (At3g50670), ribosomal protein $S 21$ family protein (At3g26360), RNA-binding (RRM/RBD/RNP motifs) (At3g04500), MCM10 (At2g20980), prenylated RAB acceptor 1.E (At1g08770), defensin (At5g33355), Solanum lycopersicum SlSmDla (Solyc06g084310.2.1; MT598822) and SlSmD1b (Solyc09g064660.2.1; MT598823). The transcriptome data are available at the Sequence Read Archive (SRA) via accession numbers PRJDB5797 (A. thaliana galls at 5 and 7 dpi with M. incognita and noninoculated roots; Yamaguchi et al., 2017) and GSE153171 (A. thaliana Col-0/ P35S:MiEFF18/ smd1b roots).

\section{Results}

\section{MiEFF18 is a secreted effector that localises to the nucleoplasm and nucleolus of plant} cells

MiEFF18 is a putative M. incognita secreted effector encoded by the Minc18636 gene. Minc18636 and its paralog, Minc15401, are more strongly expressed at the juvenile parasitic stages than at the J2 pre-parasitic stage, and are specifically expressed in the subventral oesophageal glands (SvG) of both pre-parasitic and parasitic juveniles (Rutter et al., 2014; Nguyen et al., 2018). MiEFF18 displays no similarity to any sequences out of the genus Meloidogyne or motifs included in public databases. MiEFF18 is a 312-amino acid (aa) protein with a signal peptide for secretion (aa 1 to 21, according to the Phobius prediction tool (Käll et al., 2007), an N-terminal region rich in aspartic acid and glutamic acid (D-E; 55\%) and a C-terminal region enriched in lysine (K; 40\%) (Fig. 1a; Fig. S1). In silico assays predicted the presence of several nuclear localisation signals (NLS) and one nucleolar localisation signal (NoLS) in MiEFF18 (Fig. 1a), suggesting that this protein would be imported into the nuclei of host plant cells. We produced specific antibodies against the complete MiEFF18 protein in E. coli, to check that this protein was, indeed, secreted in planta 
(Fig. S2a-c). As expected, immunolocalisation experiments on pre-parasitic J2s showed the MiEFF18 to be present in the two SvGs (Fig. 1b-e), consistent with published in situ hybridisation results (Rutter et al., 2014; Nguyen et al., 2018). Within the SvGs and their secretory tracks, MiEFF18 localised with punctate structures corresponding to secretory granules (Fig. 1c-e), consistent with its secretion during plant-nematode interactions. No signal was observed, with the exception of a non-specific signal, with the pre-immune serum in pre-parasitic J2s (Fig. S2b-c). To demonstrate secretion of MiEFF18 in planta, we used affinity-purified antibodies to immunolocalise MiEFF18 on gall sections (Fig. S2d). MiEFF18 production occurs in the SvGs of parasitic juveniles (Fig. 1f). In galls, we detected MiEFF18 in giant cells where it accumulated in the nuclei (Fig. 1g-h'), confirming its injection within the host cells during $M$. incognita parasitism. No signal was detected within the giant cells when using only the Alexa Fluor 488-conjugated secondary antibody (Fig. S2e-f). These results provide evidence for the secretion of MiEFF18 in planta during parasitism and its targeting to the plant cell nucleus.

\section{MiEFF18 interacts with the spliceosomal ribonucleoprotein SmD1}

We investigated the effector function of MiEFF18, by performing a yeast two-hybrid (Y2H) screen to search for interactors in tomato. In this system, we used MiEFF18 without its signal peptide as a bait, and a tomato root cDNA library from healthy and $M$. incognita-infected roots (Hybrigenics) as the prey. We screened 48.5 million interactions between MiEFF18 and proteins encoded by the cDNA library. We identified one major target, a ribonucleoprotein, SmD1, which was captured 26 times, whereas other candidates were captured only one to four times (Fig. S3). There are two genes encoding SmD1 proteins with $100 \%$ aa identity in Solanum lycopersicum (SlSmD1a_Solyc06g084310 and SlSmD1b_Solyc09g064660). Using a pairwise $\mathrm{Y} 2 \mathrm{H}$ approach, we independently validated the interaction between the full-length sequences of MiEFF18 and S1SmD1 (Solyc06g084310) (Fig. 2a). As a control, we investigated the interaction between S1SmD1 and another M. incognita effector, MiEFF16, encoded by the Minc16401 gene, expressed in the subventral glands, and also localising to the nucleoplasm and the nucleolus of plant cells following transient expression in Nicotiana benthamiana leaves (Fig. S4). No interaction was observed between MiEFF16 and S1SmD1 in yeast (Fig. 2a).

We investigated the colocalisation of MiEFF18 and its target, SmD1, in plant cells, by transiently expressing constructs encoding RFP-MiEFF18 and the GFP-SISmD1 fusion 
proteins in $N$. benthamiana. We confirmed the colocalisation of MiEFF18 and S1SmD1 in the nucleoplasm and nucleolus (Fig. 2b). S1SmD1 was also localised in nucleoplasmic speckles, whereas MiEFF18 was not detected in these structures (Fig. 2b). We used a bimolecular fluorescent complementation (BiFC) assay for the validation and localisation in planta of the interaction between MiEFF18 and S1SmD1. Using three combinations of BiFC vectors, we showed that MiEFF18 and SISmD1 interacted strongly in the nucleolus, with a weaker signal observed in the cytoplasm and the nucleoplasm (Fig. 2c and Fig. S5). No interaction was observed between MiEFF16 and SISmD1, with the various BiFC constructs used (Fig. S5).

Two genes, AtSmDla (At3g07590) and AtSmD1b (At4g02840), encode SmD1 proteins in A. thaliana. Using knock-out (KO) mutant lines, Elvira-Matelot et al., (2016) demonstrated that these two genes encode proteins with redundant activities, and that the smdla smdlb double mutant is lethal, as expected for a core component of the spliceosome. The smd1b single mutant displays developmental and splicing defects, whereas the smdla single mutant develops normally. AtSmD1b would account for a larger proportion of the total activity, probably due to its stronger expression in all tissues compared to AtSmDla (Elvira-Matelot et al., 2016). Using a pairwise $\mathrm{Y} 2 \mathrm{H}$ approach, we validated the interaction of the MiEFF18 effector with AtSmD1a and AtSmD1b (Fig. 2a). Overall, these results demonstrate that the MiEFF18 effector specifically interacts, in yeast and in planta, with the tomato and Arabidopsis spliceosomal SmD1 core proteins.

\section{MiEFF18 and SmD1 modulate the alternative splicing of plant genes}

As MiEFF18 interacts with SmD1, a core component of the spliceosome, we investigated the possible accumulation of similar mis-spliced transcripts in the homozygous smd $1 b$ mutant and an Arabidopsis MiEFF18-expressing line, relative to the wild type. We generated transgenic plants expressing the MiEFF18 effector under the control of a $35 \mathrm{~S}$ promoter (Fig. S6a). Noteworthy, the MiEFF18-expressing lines \#8.6 and \#13.6 exhibited a decreased susceptibility to $M$. incognita, indicating that the continued and excessive presence of MiEFF18 may be detrimental to feeding site formation (Fig. S6b). We performed RNAsequencing (RNA-seq) on total RNA isolated from the roots of 11-day-old Arabidopsis seedlings, Col-0, smd1b and MiEFF18-expressing line \#13.6. Biological triplicates were run for all samples. We then performed transcript quantification with SUPPA2, which is a computational tool that calculate relative inclusion values of alternative splicing events, based of transcript level quantification in RNA-seq data (Trincado et al., 2018; Table S2 and S3). 
The five main categories of AS events were detected: intron retention (IR), exon skipping (ES), alternative 5' splice site (A5), alternative 3' splice site (A3) and mutually exclusive exons (MX) (Fig. 3a; Fig. S7). In total, we identified 249 and 593 differential splicing events, affecting 222 and 463 genes, in the MiEFF18-expressing line and the smd1b mutant, respectively (Fig. 3a-b; Fig. S7). A high degree of overlap was observed between the two lines, with 113 AS events and 107 alternatively spliced genes common to the two lines (hypergeometric $p$ value $<4.666$ e-117; Fig. $3 b$ and Fig. S7). We also compared the dPSI corresponding to the change in each AS event in both MiEFF18-expressing lines and the $s m d 1 b$ mutants (Fig. S8). We found that the global change in AS relative to the wild-type root was significantly positively correlated in the two lines $\left(p<2 \mathrm{e}-16, \mathrm{R}^{2}=0.2406\right)$. We observed an almost perfect positive correlation if the analysis was restricted to significant differential splicing events in both lines ( $\left.p<2 \mathrm{e}-16, \mathrm{R}^{2}=0.7613\right)$. The genes concerned belonged to various families, e.g. the UDP-glucuronate decarboxylase (UXS), the heat shock protein 90 (HSP) and auxin-responsive (AUX/IAA) gene families (Table S4). The GO analysis however showed no significant enrichment in any term among the genes displaying AS in the MiEFF18-expressing line or the smd1b mutant. Using RT-qPCR, we validated an IR occurring in the MiEFF18-expressing line and the smd1b mutant in a the FAD/NAD $(P)$ binding oxidoreductase (At5g11330) gene, and an A3 event in RNA-binding protein (At3g04500), an IR in the ribosomal protein S21 family protein (At3g26360) and an A5 event in U1 snRNP 70K (At3g50670) genes occurring in the smd1b mutant (Fig. 3e; Fig.S9). Thus, MiEFF18 can modulate AS through SmD1, as the ectopic expression of MiEFF18 partially mimics the global change in AS pattern observed in the $s m d 1 b$ mutant line.

\section{M. incognita triggers alternative splicing during giant cell formation}

We used available RNA-seq data from Arabidopsis galls at 5 and 7 dpi and from noninoculated Col-0 roots (Yamaguchi et al., 2017) to investigate AS events during giant cell formation in Arabidopsis. SUPPA analysis identified 411 and 443 genes that underwent AS in response to $M$. incognita infection at 5 and $7 \mathrm{dpi}$, respectively (Fig.3a; Table S5 and S6). In total, 701 genes were alternatively spliced at either 5 or 7 dpi (Fig. 3c), representing 840 different AS events (Fig. S7d). GO analysis on these 701 AS genes revealed highly significant enrichment in the term "post embryonic development" ( $p$-value=5.8e-07), including 10 EMBRYO DEFECTIVE (EMB) genes (EMB 1353, EMB1995/ATS2, EMB1629/APO2, EMB 2728/RPE, EMB76/DCL1, EMB1006, EMB1379, EMB2768, EMB1401/EIF2 BETA and 
$E M B 1796 / N U W A$ ) and genes involved in hormone signalling (e.g. the gibberellin receptor GA INSENSITIVE DWARF1C, the cytokinin receptor WOODEN LEG (WOL/CRE1) and the auxin-responsive IAA28). In addition we noticed an enrichment in GO terms "nucleotide binding" $(p$-value=2.6e-05), "single-stranded DNA binding" $(p$-value=5.6e-05) and "ribonucleotide binding" ( $p$-value=5.1e-04) (Table S7). These results provide a first insight into the importance of AS as a regulatory mechanism involved in giant cell formation.

We then investigated whether the modulation of SmD1 function by the MiEFF18 effector could account for the AS observed upon RKN infection. Interestingly, 34.2\% (76 genes) and $24.8 \%$ (115 genes) of the genes displaying AS changes in the MiEFF18-expressing line and in the smdlb mutant, respectively, were also affected at 5 or 7 dpi with $M$. incognita; this corresponds to significant enrichment (hypergeometric $p$-value <2.0e-61) (Fig. 3d). In total, 39 of the genes displaying AS were common to the three sets of conditions, suggesting that the MiEFF18 effector and SmD1 may be at least partly responsible for the AS occurring in roots in response to RKN infection. These genes included those involved in hormone signalling, such as the auxin-responsive IAA27, the CALCIUM-DEPENDENT PROTEIN KINASE 4 (CPK4) involved in ABA signalling, and genes encoding RNA-binding proteins, such as GLYCINE-RICH RNA-BINDING PROTEIN 2 (ATGRP2) or NUCLEAR TRANSPORT FACTOR 2 (NTF2). Thus MiEFF18 could account for AS triggered in Arabidopsis following infection with $M$. incognita to modulate giant cell proteome.

\section{MiEFF18 and SmD1 modulate expression of plant genes involved in giant cell formation} Using RNA-seq data, we also identified 511 and 1,160 differentially expressed genes (DEGs) in the Arabidopsis MiEFF18-expressing line and the homozygous smd1b mutant, respectively, relative to wild-type Col-0 plants (Fig. 4a-c; Table S8 and S9). We found that 187 DEGs (130 upregulated and 57 downregulated genes) were common to MiEFF18expressing and $s m d 1 b$ plants. Interestingly, $38.0 \%$ of the DEGs in the MiEFF 18 -expressing line and the smdlb mutant were also differentially expressed at 5 and/or 7 dpi with $M$. incognita (Fig. 4b-c; Fig. S10; Table S10 and S11). RT-qPCR was used to confirm the RNAseq data (Fig. 4d). We validated the upregulation of the DNA replication-related MCM10 (At2g20980) gene and the downregulation of the Prenylated RAB acceptor 1.E (At1g08770) and a defensin (At5g33355) genes in the MiEFF18-expressing line and/or the smdlb mutant, relative to Col-0. These results are consistent with the modulation of plant gene expression by MiEFF18, through interaction with the SmD1 protein. A GO term analysis highlighted an 
overrepresentation of genes involved in "microtubule-based movement" ( $p$-value=9.1e-25) and "cell cycle process" ( $\mathrm{p}$-value $=4.9 \mathrm{e}-8)$ in the MiEFF18-expressing line, whereas GO terms associated with "plant-type cell wall organization" (p-value=1.1e-05), "response to stimulus" $(p-v a l u e=4.1 \mathrm{e}-05)$ and "response to oxidative stress" ( $p$-value $=4.1 \mathrm{e}-05)$ were overrepresented in the smd1b mutant (Fig. 4e, Fig. S11, Table S7). Interestingly, four GO terms were overrepresented in all three sets of conditions: "cytoskeleton organization", "cytoskeletal protein binding", "microtubule binding" and "tubulin binding" (Fig. 4e, Table S7).

\section{$A t S m D 1 b$ is instrumental to root knot nematode parasitism}

We investigated the possible role of Sm proteins, and SmD1 in particular, in RKN parasitism. We began by browsing transcriptomic data to determine whether the expression of $S m$ genes in galls was induced by $M$. incognita infection. Genes encoding the core Sm protein components of the spliceosome, including AtSmDla, are generally induced upon infection (Table S12), suggesting a possible role in the plant-nematode interaction. We investigated the function of Arabidopsis AtSmDl genes during parasitism further, by inoculating the smdla and smd1b Arabidopsis knockout mutants (Elvira-Matelot et al., 2016) with M. incognita J2s. Inoculation resulted in a mean decrease of $30 \%$ in the number of females producing egg masses in smdlb plants relative to wild-type Col-0 (Fig. 5a). Inoculation had no significant effect on the number of females producing egg masses in smdla plants. This result is consistent with AtSmD1b being strongly expressed in Arabidopsis, whereas AtSmDla is not (Elvira-Matelot et al., 2016). We investigated whether the giant cells formed on the smd1b plants displayed developmental defects. We observed these giant cells directly, under a confocal microscope, after BABB clearing. A comparison of the mean surface areas of the giant cells in each gall showed that giant cells from smdlb plants were 37\% smaller than those from control plants (Fig. 5b and 5c). Thus, the AtSmD1b protein plays an important role in the formation of giant cells and is required for successful nematode infection.

\section{Discussion}

\section{MiEFF18 interacts with a nuclear spliceosomal protein}

Meloidogyne spp. are among the most devastating plant pathogens, but our understanding of the molecular basis of RKN pathogenicity remains limited. RKN secrete hundreds of effectors, enabling them to overcome host defences and to induce the redifferentiation of root 
cells into permanent feeding cells. However, the functions of most of these effectors remain to be determined (Mitchum et al., 2013; Truong et al., 2015; Vieira \& Gleason, 2019; Mejias et al., 2019). One of the predicted secreted effectors, MiEFF18, has been shown to be specifically overexpressed within the nematode subventral oesophageal glands at an early stage of parasitism (Rutter et al., 2014; Nguyen et al., 2018).

We showed, by immunolocalisation studies on J2s, that MiEFF18 was present in secretory granules in the subventral gland cells. In plant-parasitic nematodes, these structures are thought to be involved in the delivery of secretions from the oesophageal glands to the stylet, through which they are secreted into the host tissues (Sundermann \& Hussey, 1988; Hussey \& Mims, 1990; Wang et al., 2010). Immunolocalisation on gall sections further demonstrated MiEFF18 secretion within the giant cells, where it accumulated in the nuclei, validating the in silico-predicted nuclear localisation of this effector in planta. Secretion has been demonstrated experimentally for very few effectors, and even fewer have been shown to be delivered to the giant cells. M. incognita MiMIF-2 (Zhao et al., 2019a) was localised in the cytoplasm, whereas the other effectors (Mi-EFF1, MjNULG1a, MgGPP and Mg16820) were immunolocalised in giant cell nuclei (Jaouannet et al., 2012; Lin et al., 2012; Chen et al., 2017b; Naalden et al., 2018). Our findings support the notion that the nucleus is a key cellular compartment that must be targeted by the parasite, for the regulation of nuclear processes essential for giant cell development, such as cell cycle regulation and transcription (Hewezi \& Baum, 2013; Quentin et al., 2013).

Using a $\mathrm{Y} 2 \mathrm{H}$ screen, we identified the nuclear spliceosomal SmD1 protein as a potential target of MiEFF18. SmD1, together with six other small ribonucleoprotein particle (Sm) proteins $(\mathrm{SmB}, \mathrm{SmD} 2, \mathrm{SmD} 3, \mathrm{SmE}, \mathrm{SmF}$ and $\mathrm{SmG})$, forms a heptameric ring structure surrounding the U-rich small nuclear RNAs (snRNAs) (Matera \& Wang, 2014). These snRNP complexes are core components of the spliceosome and play a key role in pre-mRNA splicing (i.e. the correct removal of introns from pre-RNA). When the Sm ring is assembled on the different snRNA molecules in the cytoplasm, it can enter the nucleus, where it initially accumulates in Cajal bodies, and finally, the fully assembled spliceosome executes splicing in the nucleoplasm and, more specifically, in nuclear speckles. Thus, in plants, SmD1 may localise to the nucleoplasm, nucleolus, nuclear speckles, Cajal bodies and cytoplasm, consistent with previous reports (Pendle et al., 2005; Fujioka et al., 2007; Elvira-Matelot et al., 2016; Huertas et al., 2019). We validated the localisation of SmD1 in the cytoplasm and the nucleus, where it could interact with MiEFF18. 


\section{Ectopic MiEFF18 expression mimics the effect of SmD1 impairment on AS}

539 The finding that the ectopic expression of MiEFF18 in planta mimics characteristics of the $s m d 1 b$ mutation provides further evidence in favour of SmD1 being the target of MiEFF18. AtSmD1b has recently been shown to modulate the AS of specific transcripts (Elvira-Matelot et al., 2016). In Arabidopsis, $70 \%$ of the genes may be alternatively spliced, and AS has been shown to play a significant role in plant development and responses to abiotic stresses (Reddy et al., 2013; Staiger and Brown, 2013). AS provides a layer of genetic regulation mediating rapid responses to different stimuli by increasing proteomic diversity. It can affect the stability of a transcript, particularly if the 5'UTR or 3'UTR is concerned. It can also lead to a loss/gain of protein function if the open reading frame is modified, by a frameshift or the creation of a new premature stop codon (Chaudhary et al., 2019). Only a few studies to date have focused on plant Sm proteins. They investigated the Arabidopsis SmD3 (Swaraz et al., 2011) and SmE (Huertas et al., 2019) proteins, and data are also available for the Sm-Like protein LSm8, another core component of the spliceosome (Carrasco-López et al., 2017). Genome-wide AS analysis has confirmed the role of SmE and LSm8 in regulating AS in Arabidopsis, enabling plants to adapt to unfavourable abiotic environments. We expand here, by a transcriptomic approach, the role of AtSmD1b in regulating AS, and we reveal its crucial function in a biotic interaction. Our RNAseq data showed that MiEFF18 could coordinate this AtSmD1b function during RKN parasitism. Indeed, half of the splicing events, in 107 genes, induced by the ectopic expression of MiEFF18 in Arabidopsis, were also induced by AtSmD1b mutation, suggesting that MiEFF18 controls susceptibility to RKN by directly modulating the host cell transcriptome.

\section{Alternative splicing occurs upon RKN parasitism in Arabidopsis}

AS may play an important role in plant responses to pathogens (Rigo et al., 2019). Very few studies have reported the AS events occurring in plants in response to infection with bacterial, viral or fungal pathogens (Howard et al., 2013; Mandadi \& Scholthof, 2015; Rubio et al., 2015; Song et al., 2017; Zheng et al., 2017; Bedre et al., 2019; Ma et al., 2019; Zhang et al., 2019; Wang et al., 2020). Specific AS events occur in plants in response to a pathogen. A number of different, specific splice variants have, for example, been shown to accumulate in wheat in response to infection with two fungal pathogens, Blumeria graminis f. sp. tritici and 
specificity of the AS of pre-mRNA and controlling stress responses remain poorly understood (Catalá et al., 2019). We provide here a transcriptome-wide description of the AS events occurring in galls 5 and 7 dpi with M. incognita. We show that, in galls, AS genes exhibited significant alternative 3' splice site selection rather than intron retention, which is usually predominant in plant response to stress (Laloum et al., 2018). In addition, in galls AS occurs in genes specifically related to giant cell ontogenesis. Indeed, we show enrichment in genes related to post-embryonic organogenesis among the genes displaying AS in galls. The developmental reprogramming required for giant cell formation involves modulation of the expression of genes involved in root cell identity and root development (Yamaguchi et al., 2017; Olmo et al., 2020). The Mi16D10 effector has been shown to manipulate two of these proteins, both of which are SCARECROW-like transcription factors regulating gene expression during root organogenesis (Huang et al., 2006). Our results suggest that MiEFF18, by interfering with AtSmD1b function, may affect these processes in a broader manner, providing transcriptional control over several of these genes.

Recently, effectors have been shown to interfere with the plant spliceosome machinery. The PsAvr3c effector, secreted by the plant pathogenic oomycete Phytophthora sojae, has been shown to interfere with the soybean serine/lysine/arginine-rich protein GmSKRP1, modifying the pattern of AS in the host plant to subvert immunity (Huang et al., 2017). Similarly, the H. schachtii 30D08 effector has been shown to interact with the Arabidopsis SMU2 auxiliary spliceosomal protein. The 30D08 protein allows the cyst nematode to alter pre-mRNA splicing and the expression of genes involved in feeding site development (Verma et al., 2018). We can, thus, hypothesize that, acting through its interaction with a core spliceosomal protein, MiEFF18 modulates the AS occurring in giant cells upon plant-RKN interaction.

\section{MiEFF18 and SmD1 regulate the expression of genes involved in giant cell ontogenesis}

A broad reprogramming of transcription occurs upon RKN infection, as already demonstrated in many plants, including Arabidopsis (Escobar et al., 2011; Favery et al., 2016; Yamaguchi et al., 2017). Thousands of plant genes involved in diverse processes, including cell cycle activation, cell wall modification, and hormone and defence responses, are differentially expressed during RKN parasitism (Favery et al., 2016). Ectopic expression of MiEFF18 and partial impairment of SmD1 activity (using the $s m d 1 b$ mutant) had similar effects on the expression of various genes differentially expressed upon $M$. incognita infection and giant cell 
603

604

605

606

607

608

609

610

611

612

613

614

615

616

617

618

619

620

621

622

623

624

625

626

627

628

629

630

631

632

633

634

formation in Arabidopsis. In particular, genes involved in DNA replication (e.g. the MCM gene family), in DNA repair and in microtubule network regulation (e.g. encoding kinesins or the MAP65 proteins), or encoding proteins involved in spindle assembly (MAP70-1; IQ DOMAIN 31; TPX2) were upregulated in the Arabidopsis lines studied here. This finding is consistent with the synchronised activation of cell cycle processes, such as acytokinetic mitoses and DNA amplification, that occurs during giant cell formation (De Almeida Engler \& Gheysen, 2013; Favery et al., 2016). Deregulation of the expression of key regulators of the cell cycle and of cytoskeleton regulators through mutations (e.g. map65-3 or wee1.1), or ectopic expression (e.g. Kip-Related Protein (KRP)-expressing lines), leads to defective giant cell development (Caillaud et al., 2008; Coelho et al., 2017; Vieira \& de Almeida Engler, 2017; Cabral et al., 2020).

We show here that constitutive expression of the MiEFF18 effector decreases the susceptibility of Arabidopsis to M. incognita. However, the ectopic expression of MiEFF18 may not reflect what happens under physiological conditions in a giant cell, where the effector must be timely delivered in a precise amount. The excess of some effectors in plants may modify plant physiology and cell function, and confer plant resistance to biotic and/or abiotic stresses. Such observations could be made when expressing in planta oomycete effectors (e.g. PsCRN161 or PsCRN115; Rajput et al. 2015) or cyst nematode effectors (e.g. Hs32E03 and Hs30D08; Vijayapalani et al. 2018 and Verma et al. 2018). In addition, the partial impairement of SmD1 function affects the susceptibility of Arabidopsis to RKN, impacting giant cell development. Alltogether our results demonstrate that MiEFF18 effector interacts with AtSmD1b and may perturbate its homeostasis to facilitate the de novo formation of the giant feeding cells unique to RKN parasitism, by regulating key developmental processes.

The answer on how the EFF18 effector manipulates the SmD1 function may come from an analysis of the structure of the MiEFF18. The K-rich C-terminal part of the effector, carrying NLS and NoLS, undoubtedly mediates import into the nucleus, and the N-terminal part of the molecule carries D/E repeats, which are often found in DNA/RNA mimic proteins (Chou \& Wang, 2015). These proteins regulate the activity of various DNA/RNA-binding proteins involved in diverse nuclear processes, such as chromatin assembly, DNA repair or transcriptional regulation (Chou \& Wang, 2015; Wang et al., 2019). Further studies of this effector-target pair and associated RNAs would improve our understanding of the role and regulation of the spliceosome machinery in plants and might lead to the development of 
635

636

637

638

639

640

641

642

643

644

645

646

647

648

649

650

651

652

653

654

655

656

657

658

659

660

661

662

663

664

665

666

667

applications in new control strategies based on the loss of a susceptibility gene essential for development of the disease.

\section{Acknowledgments}

We thank Hybrigenics Services (Paris, France) for providing the pB27 and pP6 vectors and the L40 4 Gal4 and Y187 yeast strains, and Syngenta for MiEFF18 production. We thank Dr Laurent Deslandes (LIPM, Castanet Tolosan, France) for helpful discussions. Microscopy work was performed at the SPIBOC imaging facility of the Institut Sophia Agrobiotech. We thank Dr Olivier Pierre and the whole platform team for their help with microscopy. We thank Dr Lucie Monticelli for contributing statistical analysis. We thank Dr Janice de Almeida Engler for valuable advice on immunolocalisation. This work was funded by the INRA SPE department and the French Government (National Research Agency, ANR) through the 'Investments for the Future' LabEx SIGNALIFE: programme reference \#ANR-11-LABX0028-01, by the INRA-Syngenta Targetome project, by the French-Japanese bilateral collaboration programmes PHC SAKURA 2016 \#35891VD and 2019 \#43006VJ and by the French-Chinese bilateral collaboration program PHC XU GUANGQI 2020 \#45478PF. This work was also supported by the Agence Nationale de la Recherche ANR-16-CE12-0032 (SPLISIL to HV and MC) and the "Laboratoire d'Excellence (LABEX)" Saclay Plant Sciences (SPS; ANR-10-LABX-40). J.M. holds a doctoral fellowship from the French Ministère de l'Enseignement Supérieur, de la Recherche et de l'Innovation (MENRT grant). N.M.T. was supported by a USTH fellowship, as part of the 911-USTH programme of the Ministry of Education and Training of The Socialist Republic of Vietnam. Y.P.C. got scholarships from China Scholarship Council (No. 201806350108) for studying at INRAE, France.

\section{Author contributions}

J.M. designed and performed experiments, and interpreted results; J.M., Y.P.C. and N.M.T. performed yeast two-hybrid assays and generated constructs; SW, J.B. and M.D.C. performed the transcriptome analysis and analysed AS data; H.V. and N.B. contributed material and analysed the data; N.M. produced the nematodes and tomato plants; J.M., J.B., H.V., P.A., B.F. and M.Q. wrote the article; P.A., B.F. and M.Q. obtained funding, designed the work and supervised the experiments and data analyses; all the authors read and edited the article. 
668

669

670

671

672

673

674

675

676

677

678

679

680

681

682

683

684

685

686

687

688

689

690

691

692

693

694

695

696

697

698

699

700

\section{References}

de Almeida Engler J, Favery B. 2011. The Plant Cytoskeleton Remodelling in Nematode Induced Feeding Sites. In: Genomics and Molecular Genetics of Plant-Nematode Interactions. Dordrecht: Springer Netherlands, 369-393.

de Almeida Engler J, Gheysen G. 2013. Nematode-induced endoreduplication in plant host cells: why and how? Molecular Plant-Microbe Interactions 26: 17-24.

Barnes SN, Wram CL, Mitchum MG, Baum TJ. 2018. The plant-parasitic cyst nematode effector GLAND4 is a DNA-binding protein. Molecular Plant Pathology 19: 2263-2276.

Bartlem DG, Jones MGKK, Hammes UZ. 2014. Vascularization and nutrient delivery at root-knot nematode feeding sites in host roots. Journal of Experimental Botany 65: 17891798.

Bedre R, Irigoyen S, Schaker PDC, Monteiro-Vitorello CB, Da Silva JA, Mandadi KK. 2019. Genome-wide alternative splicing landscapes modulated by biotrophic sugarcane smut pathogen. Scientific Reports 9: 1-12.

Bent AF, Clough SJ. 1998. Agrobacterium Germ-Line Transformation: Transformation of Arabidopsis without Tissue Culture. In: Plant Molecular Biology Manual. Springer Netherlands, 17-30.

Cabral D, Banora MY, Antonino JD, Rodiuc N, Vieira P, Coelho RR, Chevalier C, Eekhout T, Engler G, De Veylder L, et al. 2020. The plant WEE1 kinase is involved in checkpoint control activation in nematode-induced galls. New Phytologist 225: 430-447. Cabrera J, Bustos R, Favery B, Fenoll C, Escobar C. 2014. NEMATIC: A simple and versatile tool for the insilico analysis of plant-nematode interactions. Molecular Plant Pathology 15: 627-636.

Cabrera J, Olmo R, Ruiz-Ferrer V, Abreu I, Hermans C, Martinez-Argudo I, Fenoll C, Escobar C. 2018. A Phenotyping Method of Giant Cells from Root-Knot Nematode Feeding Sites by Confocal Microscopy Highlights a Role for CHITINASE-LIKE 1 in Arabidopsis. International Journal of Molecular Sciences 19: 429.

Caillaud M-C, Abad P, Favery B. 2008. Cytoskeleton reorganization. Plant Signaling \& Behavior 3: 816-818.

Caillaud M-C, Favery B. 2016. In Vivo Imaging of Microtubule Organization in Dividing Giant Cell. In: Caillaud M-C, ed. Methods in Molecular Biology. New York: Springer New York, 137-144.

Caillaud M-C, Paganelli L, Lecomte P, Deslandes L, Quentin M, Pecrix Y, Le Bris M, 
Marfaing N, Abad P, Favery B. 2009. Spindle Assembly Checkpoint Protein Dynamics Reveal Conserved and Unsuspected Roles in Plant Cell Division. PLoS ONE 4: e6757. Carrasco-López C, Hernández-Verdeja T, Perea-Resa C, Abia D, Catalá R, Salinas J. 2017. Environment-dependent regulation of spliceosome activity by the LSM2-8 complex in Arabidopsis. Nucleic Acids Research 45: 7416-7431.

Catalá R, Carrasco-López C, Perea-Resa C, Hernández-Verdeja T, Salinas J. 2019.

Emerging roles of $1 \mathrm{sm}$ complexes in posttranscriptional regulation of plant response to abiotic stress. Frontiers in Plant Science 10: 167.

Chaudhary S, Khokhar W, Jabre I, Reddy ASN, Byrne LJ, Wilson CM, Syed NH. 2019. Alternative splicing and protein diversity: Plants versus animals. Frontiers in Plant Science 10: 708 .

Chen J, Lin B, Huang Q, Hu L, Zhuo K, Liao J. 2017. A novel Meloidogyne graminicola effector, MgGPP, is secreted into host cells and undergoes glycosylation in concert with proteolysis to suppress plant defenses and promote parasitism. PLoS Pathogens 13: e1006301.

Chou CC, Wang AHJ. 2015. Structural D/E-rich repeats play multiple roles especially in gene regulation through DNA/RNA mimicry. Molecular BioSystems 11: 2144-2151.

Coelho RR, Vieira P, Antonino de Souza Júnior JD, Martin-Jimenez C, De Veylder L, Cazareth J, Engler G, Grossi-de-Sa MF, de Almeida Engler J. 2017. Exploiting cell cycle inhibitor genes of the KRP family to control root-knot nematode induced feeding sites in plants. Plant Cell and Environment 40: 1174-1188.

Deslandes L, Rivas S. 2011. The plant cell nucleus. Plant Signaling \& Behavior 6: 42-48. Elvira-Matelot E, Bardou F, Ariel F, Jauvion V, Bouteiller N, Le Masson I, Cao J, Crespi MD, Vaucheret H. 2016. The Nuclear Ribonucleoprotein SmD1 Interplays with Splicing, RNA Quality Control, and Posttranscriptional Gene Silencing in Arabidopsis. The Plant Cell 28: 426-438.

Escobar C, Brown S, Mitchum M. 2011. Transcriptomic and Proteomic Analysis of the Plant response to Nematode Infection. In: Jones J, Gheysen G, Fenoll C, eds. Genomics and Molecular Genetics of Plant-Nematode Interactions. Dordrecht, Heidelberg, London \& New York: Springer, 157-173.

Favery B, Quentin M, Jaubert-Possamai S, Abad P. 2016. Gall-forming root-knot nematodes hijack key plant cellular functions to induce multinucleate and hypertrophied feeding cells. Journal of Insect Physiology. 84: 60-69. 
Fujioka Y, Utsumi M, Ohba Y, Watanabe Y. 2007. Location of a possible miRNA processing site in SmD3/SmB nuclear bodies in arabidopsis. Plant and Cell Physiology 48: $1243-1253$.

Hewezi T, Baum TJ. 2013. Manipulation of Plant Cells by Cyst and Root-Knot Nematode Effectors. Molecular Plant-Microbe Interactions 26: 9-16.

Howard BE, Hu Q, Babaoglu AC, Chandra M, Borghi M, Tan X, He L, Winter-Sederoff H, Gassmann W, Veronese P, et al. 2013. High-Throughput RNA Sequencing of Pseudomonas-Infected Arabidopsis Reveals Hidden Transcriptome Complexity and Novel Splice Variants. PLoS ONE 8: e74183.

Huang GZ, Allen R, Davis EL, Baum TJ, Hussey RS. 2006a. Engineering broad root-knot resistance in transgenic plants by RNAi silencing of a conserved and essential root-knot nematode parasitism gene. Proceedings of the National Academy of Sciences of the United States of America 103: 14302-14306.

Huang G, Dong R, Allen R, Davis EL, Baum TJ, Hussey RS. 2006b. A Root-Knot Nematode Secretory Peptide Functions as a Ligand for a Plant Transcription Factor. Mol Plant Microbe Interact 19: 463-470.

\section{Huang J, Gu L, Zhang Y, Yan T, Kong G, Kong L, Guo B, Qiu M, Wang Y, Jing M, et} al. 2017. An oomycete plant pathogen reprograms host pre-mRNA splicing to subvert immunity. Nature Communications 8: 2051.

Huertas R, Catalá R, Jiménez-Gómez JM, Castellano MM, Crevillén P, Piñeiro M, Jarillo JA, Salinas J. 2019. Arabidopsis SME1 regulates plant development and response to abiotic stress by determining spliceosome activity Specificity. Plant Cell 31: 537-554.

Hussey RS, Mims CW. 1990. Ultrastructure of esophageal glands and their secretory granules in the root-knot nematode Meloidogyne incognita. Protoplasma 165: 9-18. Jaouannet M, Perfus-Barbeoch L, Deleury E, Magliano M, Engler G, Vieira P, Danchin EGJ, Rocha M Da, Coquillard P, Abad P, et al. 2012. A root-knot nematode-secreted protein is injected into giant cells and targeted to the nuclei. New Phytologist 194: 924-931. Jaubert-Possamai S, Noureddine Y, Favery B. 2019. MicroRNAs, New Players in the Plant-Nematode Interaction. Frontiers in Plant Science 10: 1180. Jaubert S, Milac AL, Petrescu AJ, de Almeida-Engler J, Abad P, Rosso M-N. 2005. In Planta Secretion of a Calreticulin by Migratory and Sedentary Stages of Root-Knot Nematode. Molecular Plant-Microbe Interactions 18: 1277-1284.

Käll L, Krogh A, Sonnhammer ELL. 2007. Advantages of combined transmembrane 
767

768

769

770

771

772

773

774

775

776

777

778

779

780

781

782

783

784

785

786

787

788

789

790

791

792

793

794

795

796

797

798

799

topology and signal peptide prediction-the Phobius web server. Nucleic Acids Research 35: $429-432$.

Karimi M, Depicker A, Hilson P. 2007. Recombinational cloning with plant gateway vectors. Plant Physiology 145: 1144-1154.

Laloum T, Martín G, Duque P. 2018. Alternative Splicing Control of Abiotic Stress

Responses. Trends in Plant Science 23: 140-150.

Lin B, Zhuo K, Wu P, Cui R, Zhang L-H, Liao J. 2012. A Novel Effector Protein, MJNULG1a, Targeted to Giant Cell Nuclei Plays a Role in Meloidogyne javanica Parasitism . Molecular Plant-Microbe Interactions 26: 55-66.

Ma JQ, Wie LJ, Lin A, Zhang C, Sun W, Yang B, Lu K, Li JN. 2019. The alternative splicing landscape of brassica napus infected with leptosphaeria maculans. Genes 10: 296. Mandadi KK, Scholthof KBG. 2015. Genome-wide analysis of alternative splicing landscapes modulated during plant-virus interactions in brachypodium distachyon. Plant Cell 27: 71-85.

Matera AG, Wang Z. 2014. Erratum: A day in the life of the spliceosome (Nature Reviews Molecular Cell Biology (2014) 15 (108-122)). Nature Reviews Molecular Cell Biology 15: 294.

Mejias J, Truong NM, Abad P, Favery B, Quentin M. 2019. Plant Proteins and Processes Targeted by Parasitic Nematode Effectors. Frontiers in Plant Science 10: 970.

Mitchell A, Chang HY, Daugherty L, Fraser M, Hunter S, Lopez R, McAnulla C, McMenamin C, Nuka G, Pesseat S, et al. 2015. The InterPro protein families database: The classification resource after 15 years. Nucleic Acids Research 43: D213-D221.

Mitchum MG, Hussey RS, Baum TJ, Wang X, Elling AA, Wubben M, Davis EL. 2013.

Nematode effector proteins: An emerging paradigm of parasitism. New Phytologist 199: 879894.

Motion GB, Amaro TMMM, Kulagina N, Huitema E. 2015. Nuclear processes associated with plant immunity and pathogen susceptibility. Briefings in Functional Genomics 14: 243252.

Naalden D, Haegeman A, de Almeida-Engler J, Birhane Eshetu F, Bauters L, Gheysen G. 2018. The Meloidogyne graminicola effector $\operatorname{Mg} 16820$ is secreted in the apoplast and cytoplasm to suppress plant host defense responses. Molecular Plant Pathology 19: 24162430.

Nguyen C-N, Perfus-Barbeoch L, Quentin M, Zhao J, Magliano M, Marteu N, Da Rocha 
800

801

802

803

804

805

806

807

808

809

810

811

812

813

814

815

816

817

818

819

820

821

822

823

824

825

826

827

828

829

830

831

832

M, Nottet N, Abad P, Favery B. 2018. A root-knot nematode small glycine and cysteine-rich secreted effector, MiSGCR1, is involved in plant parasitism. New Phytologist 217: 687-699.

Olmo R, Cabrera J, Díaz-Manzano FE, Ruiz-Ferrer V, Barcala M, Ishida T, García A, Andrés MF, Ruiz-Lara S, Verdugo I, et al. 2020. Root-knot nematodes induce gall formation by recruiting developmental pathways of post-embryonic organogenesis and regeneration to promote transient pluripotency. New Phytologist 227: 200-215.

Pendle AF, Clark GP, Boon R, Lewandowska D, Lam YW, Andersen J, Mann M, Lamond AI, Brown JWS, Shaw PJ. 2005. Proteomic Analysis of the Arabidopsis Nucleolus Suggests Novel Nucleolar Functions $\square$ D. Molecular Biology of the Cell 16: 260-269.

Postnikova OA, Hult M, Shao J, Skantar A, Nemchinov LG. 2015. Transcriptome analysis of resistant and susceptible alfalfa cultivars infected with root-knot nematode Meloidogyne incognita. PloS one. 10(3): e0123157.

Quentin M, Abad P, Favery B. 2013. Plant parasitic nematode effectors target host defense and nuclear functions to establish feeding cells. Frontiers in Plant Science 4: 53.

Rancurel C, van Tran T, Elie C, Hilliou F. 2019. SATQPCR: Website for statistical analysis of real-time quantitative PCR data. Molecular and Cellular Probes 46: 101418.

Reddy ASN, Marquez Y, Kalyna M, Barta A. 2013. Complexity of the Alternative Splicing Landscape in Plants. The Plant Cell 25: 3657-3683.

Rigo R, Bazin J, Crespi M, Charon C. 2019. Alternative Splicing in the Regulation of Plant-Microbe Interactions. Plant and Cell Physiology 60: 1906-1916.

Rubio M, Rodríguez-Moreno L, Ballester AR, de Moura MC, Bonghi C, Candresse T, Martínez-Gómez P. 2015. Analysis of gene expression changes in peach leaves in response to Plum pox virus infection using RNA-Seq. Molecular Plant Pathology 16: 164-176.

\section{Rutter WB, Hewezi T, Abubucker S, Maier TR, Huang G, Mitreva M, Hussey RS,} Baum TJ. 2014. Mining Novel Effector Proteins from the Esophageal Gland Cells of Meloidogyne incognita. Molecular plant-microbe interactions 27: 965-974.

Shukla N, Yadav R, Kaur P, Rasmussen S, Goel S, Agarwal M, Jagannath A, Gupta R, Kumar A. 2018. Transcriptome analysis of root-knot nematode ( Meloidogyne incognita )infected tomato ( Solanum lycopersicum ) roots reveals complex gene expression profiles and metabolic networks of both host and nematode during susceptible and resistance responses. Molecular Plant Pathology 19: 615-633.

Singh SK, Hodda M, Ash GJ. 2013. Plant-parasitic nematodes of potential phytosanitary importance, their main hosts and reported yield losses. EPPO Bulletin 43: 334-374. 
Song J, Liu H, Zhuang H, Zhao C, Xu Y, Wu S, Qi J, Li J, Hettenhausen C, Wu J. 2017. Transcriptomics and Alternative Splicing Analyses Reveal Large Differences between Maize Lines B73 and Mo17 in Response to Aphid Rhopalosiphum padi Infestation. Frontiers in Plant Science 8: 1738.

Staiger D, Brown JWS. 2013. Alternative splicing at the intersection of biological timing, development, and stress responses. Plant Cell 25: 3640-3656.

Sundermann CA, Hussey RS. 1988. Ultrastructural Cytochemistry of Secretory Granules of Esophageal Glands of Meloidogyne incognita. Journal of nematology 20: 141-149.

Swaraz AM, Park YD, Hur Y. 2011. Knock-out mutations of Arabidopsis SmD3-b induce pleotropic phenotypes through altered transcript splicing. Plant Science 180: 661-671. Trincado JL, Entizne JC, Hysenaj G, Singh B, Skalic M, Elliott DJ, Eyras E. 2018. SUPPA2: Fast, accurate, and uncertainty-aware differential splicing analysis across multiple conditions. Genome Biology 19: 40.

Truong NM, Nguyen C-N, Abad P, Quentin M, Favery B. 2015. Function of Root-Knot Nematode Effectors and Their Targets in Plant Parasitism. In: Escobar C, Fenoll C, eds. Advance in Botanical Research: Plant Nematode Interactions A View on Compatible Interrelationships. Academic Press, 293-324. Verma A, Lee C, Morriss S, Odu F, Kenning C, Rizzo N, Spollen WG, Lin M, McRae AG, Givan SA, et al. 2018. The novel cyst nematode effector protein 30D08 targets host nuclear functions to alter gene expression in feeding sites. New Phytologist 219: 697-713. Vieira P, de Almeida Engler J. 2017. Plant cyclin-dependent kinase inhibitors of the KRP family: Potent inhibitors of root-knot nematode feeding sites in plant roots. Frontiers in Plant Science 8: 1514.

Vieira P, Gleason C. 2019. Plant-parasitic nematode effectors — insights into their diversity and new tools for their identification. Current Opinion in Plant Biology 50: 37-43. Vijayapalani P, Hewezi T, Pontvianne F, Baum TJ. 2018. An Effector from the Cyst Nematode Heterodera schachtii Derepresses Host rRNA Genes by Altering Histone Acetylation. The Plant Cell 30: tpc.00570.2018.

Wang L, Chen M, Zhu F, Fan T, Zhang J, Lo C. 2020. Alternative splicing is a Sorghum bicolor defense response to fungal infection. Planta 251: 1-13.

Wang HC, Chou CC, Hsu KC, Lee CH, Wang AHJ. 2019. New paradigm of functional regulation by DNA mimic proteins: Recent updates. IUBMB Life 71: 539-548.

Wang J, Lee C, Replogle A, Joshi S, Korkin D, Hussey R, Baum TJ, Davis EL, Wang X, 
866

867

868

869

870

871

872

873

874

875

876

877

878

879

880

881

882

883

884

885

886

887

Mitchum MG. 2010. Dual roles for the variable domain in protein trafficking and hostspecific recognition of Heterodera glycines CLE effector proteins. New Phytologist 187: 1003-1017.

Wilkinson ME, Charenton C. 2020. RNA Splicing by the Spliceosome. Annual Review of Biochemistry 89: 359-388.

\section{Yamaguchi YL, Suzuki R, Cabrera J, Nakagami S, Sagara T, Ejima C, Sano R, Aoki Y,}

Olmo R, Kurata T, et al. 2017. Root-Knot and Cyst Nematodes Activate ProcambiumAssociated Genes in Arabidopsis Roots. Frontiers in Plant Science 8: 1195.

Zhang L, Davies LJ, Elling A a. 2014. A Meloidogyne incognita effector is imported into the nucleus and exhibits transcriptional activation activity in planta. Molecular plant pathology 16(1): 48-60.

Zhang H, Mao R, Wang Y, Zhang L, Wang C, Lv S, Liu X, Wang Y, Ji W. 2019.

Transcriptome-wide alternative splicing modulation during plant-pathogen interactions in wheat. Plant Science 288: 110160.

Zhao J, Li L, Liu Q, Liu P, Li S, Yang D, Chen Y, Pagnotta S, Favery B, Abad P, et al. 2019. A MIF-like effector suppresses plant immunity and facilitates nematode parasitism by interacting with plant annexins. Journal of Experimental Botany 70: 5943-5958.

Zheng Y, Wang Y, Ding B, Fei Z. 2017. Comprehensive Transcriptome Analyses Reveal that Potato Spindle Tuber Viroid Triggers Genome-Wide Changes in Alternative Splicing, Inducible trans- Acting Activity of Phased Secondary Small Interfering RNAs, and Immune Responses Yi. 91: e002447-17. 
889

890

891

892

893

894

895

896

897

898

899

900

901

902

903

904

905

906

907

908

909

910

911

912

913

914

915

916

917

918

919

920

921

\section{Figure legends}

Fig. 1 MiEFF18 is a secreted effector that localises to the nucleus and nucleolus of plant cells. (a) Schematic diagram of the MiEFF18 protein. The predicted secretion signal peptide (SP; red box), the aspartic acid and glutamic acid (D-E)-rich region (purple box), the lysine (K)rich C-terminal region (yellow box), nuclear localisation signals (NLS) and the nucleolar localisation signal (NoLS) are shown. The NLS pat4 (KKPK, aa 235-238) and pat 7 (PAKKGKK, aa 292-298) are indicated in grey and the bipartite region (KGAAKVAKKDTKKPKD, aa 223-239) is shown in black. (b) Schematic diagram of a section through a J2. (c-e) Immunolocalisation of MiEFF18 in the subventral glands (SvGs) of M. incognita pre-parasitic J2s. (f) Immunolocalisation of MiEFF18 in the subventral glands (SvGs) of parasitic $M$. incognita. Confocal images of J2s treated with rabbit anti-MiEFF18 serum and goat anti-rabbit Alexa Fluor 488 secondary antibodies are shown. Fluorescence signals are visible in the secretory granules of the subventral glands (magnification in the insets) and in the secretory tracts (arrow). Corresponding bright-field images of the juveniles are shown in the left. Bars $=10 \mu \mathrm{m}$. m, metacorpus, $\mathrm{n}$, nucleus, SvGs, subventral glands. (gh') Localization of the secreted MiEFF18 protein in plant tissues. MiEFF18 accumulated in the giant cell nuclei. Images of Alexa Fluor 488 fluorescence, DAPI-stained nuclei and overlays are shown. (h') is an enlargement of the area framed in (h). *, giant cell. Bars $=10$ $\mu \mathrm{m}$.

Fig. 2 The MiEFF18 effector interacts with SmD1 proteins in the nucleus and nucleolus of plant cells. (a) Diploid yeasts containing the bait and prey plasmids carrying controls, effectors or SmD1 proteins (Solanum lycopersicum S1SmD1 and Arabidopsis thaliana, AtSmD1a and AtSmD1b) were spotted on plates. SD-WL corresponds to the non-selective medium without tryptophan (W) and leucine (L). Only yeasts carrying a protein-protein interaction can survive on the SD-WLH $(\mathrm{H}$, histidine $)+0.5 \mathrm{mM}$ 3-AT selective medium. Murine p53 and SV40 T-antigen T (anti T) were used as a positive control, and MiEFF16 was used as a negative control. (b) Colocalisation of RFP-MiEFF18 and GFP-S1SmD1 in $N$. benthamiana epidermal leaf cells. RFP and GFP were used as a nucleocytoplasmic control. Bars $=5 \mu \mathrm{m}$. (c) MiEFF18 and SISmD1 interact together in the nucleolus, nucleoplasm and cytoplasm in $N$. benthamiana cells. YFP fluorescence confocal images of bimolecular fluorescence complementation (BiFC) experiments with different combinations of YFPc or 
922

YFNn fused, at the C- or N-terminus, to S1SmD1 and MiEFF18, expressed in N. benthamiana epidermal cells. The MiEFF16 effector was used as a negative control. Bars $=10 \mu \mathrm{m}$.

Fig. $3 \mathrm{SmD} 1 \mathrm{~b}$ modulates alternative splicing in Arabidopsis roots. (a) Arabidopsis genes with alternative splicing (AS) events (intron retention, exon skipping, alternative 5' splice site, alternative 3' splice site, mutually exclusive exons) in the MiEFF18-expressing line and the smd $1 b$ mutant, relative to Col-0 roots, and in galls five and seven days post inoculation (dpi) with $M$. incognita, relative to uninfected Arabidopsis Col-0 roots. (b) Venn diagram showing the overlap between alternatively spliced genes in the MiEFF18-expressing line and smd1b mutant plants. (c) Venn diagram showing the overlap between alternatively spliced genes in M. incognita-induced galls at 5 and 7 dpi. (d) Venn diagram showing the overlap between genes affected in the MiEFF18-expressing line, smdlb mutant and in M. incognita-induced galls at 5 or 7 dpi. (e) Validation of the changes in AS pattern detected in the roots of Arabidopsis MiEFF18-expressing line, smd1b mutant and wild-type Col-0 by RT-qPCR. Data were normalised using $U B Q 10$ as a reference gene. Asterisks indicate significant differences $(* * \mathrm{P}<0.001, * * * \mathrm{P}<0.0001)$ compared to wild-type plants, as determined by $\mathrm{t}$-student test (SatqPCR software). Error bars indicate the SE. Left panels show the part of the alternately spliced genes (the black boxes represent the exons, the lines represent the introns) and the read mapping of the RNAseq (y-axis).

Fig. 4 MiEFF18 and SMD1b regulate transcript accumulation in Arabidopsis root. (a) Quantification of differentially expressed genes (DEG) in the roots of the MiEFF18expressing Arabidopsis line (EFF18) and the smd1b mutant, relative to Col-0 roots. The overlap between genes differentially expressed (up: induced; down: repressed) in the EFF18 line and the smdlb mutant is shown. (b) Venn diagram showing the overlaps between genes induced (up) in the MiEFF18-expressing line, the smd1b mutant and in M. incognita-induced galls at 5 or 7 dpi. (c) Venn diagram showing the overlaps between genes repressed (down) in the MiEFF18-expressing line, the smd $1 b$ mutant and in M. incognita-induced galls at 5 or 7dpi. (d) Validation of the expression of DEG identified in the smd1b mutant and/or the MiEFF18-expressing line, by RT-qPCR. Data were normalized against $U B Q 10$ and $O X A 1$ as constitutive genes. Asterisks indicate significant differences $(* \mathrm{P}<0.01)$ between MiEFF18expressing line or the $s m d 1 b$ mutants compared to wild-type (Col-0) plants, as determined by t-student test (SatqPCR software). Error bars indicate the SE. (e) Enrichment in GO terms for 
955

956

957

958

959

960

961

962

963

964

965

966

967

968

969

970

971

972

\section{Supporting information}

973 Table S1 Primers used in this study.

974 Table S2 Altered splicing events identified in the Arabidopsis MiEFF18-expressing line

975 Table S3 Altered splicing events identified in the Arabidopsis smd1b mutant line.

976 Table S4 Gene family (GenFam) enrichment analyses.

977 Table S5 Altered splicing events identified in Arabidopsis thaliana at 5 dpi with Meloidogyne

978 incognita.

979 Table S6 Altered splicing events identified in Arabidopsis thaliana at 7 dpi with Meloidogyne 980 incognita.

981 Table S7 Gene Ontology (GO) analyses.

982

983

984

biological processes among DEGs in the MiEFF 18-expressing line, smd1b mutant and in galls

Fig. 5 AtSmD1b is instrumental to root-knot nematode parasitism. (a) Box-and-whisker plots of females producing egg masses per plant in Col-0 control line, smdla, smd1b lines six weeks post infection with $200 \mathrm{M}$. incognita J2s. The three independent experiments are presented. The effect of plant genotype on the number of nematode egg masses was analyzed with generalized linear models (GLMs) based on a Poisson distribution, for each replicate. We used the Tukey adjustment method ('multcomp' package) for multiple testing. Different letters indicate statistically significant difference between each column. (b) Galls of Col-0 and $s m d l b$ plants collected two weeks post infection to measure the surface of the giant cells (doted line) using BABB clearing method (Cabrera et al., 2018). (c) Box-and-whisker plot of giant cell size $(\mu \mathrm{m} 2)$ measures on Col- 0 and $s m d 1 b$ plants. The impact of the plant genotype on the surface of giant cells was analysed using student $t$ test. Combined data from two independent biological replicates are shown $(\mathrm{n}=42$ and $\mathrm{n}=25)$. Significance of terms: $* * * \mathrm{P}<$ 0.001 .

Table S8 Differentially expressed genes identified in the Arabidopsis MiEFF18-expressing line

Table S9 Differentially expressed genes identified in the Arabidopsis smd1b mutant line. 
985 Table S10 Differentially expressed genes identified in Arabidopsis thaliana at 5 dpi with 986 Meloidogyne incognita.

987 Table S11 Differentially expressed genes identified in Arabidopsis thaliana at 7 dpi with 988 Meloidogyne incognita.

989 Table S12 Expression of the different Arabidopsis small nuclear ribonucleoprotein Sm core 990 genes during $M$. incognita infection.

991

992

993

994

995

996

997

998

999

1000

1001

1002

1003

1004

1005

1006

1007

1008

1009

Fig. S1 Alignment of the Minc18636 and Minc15401 proteins.

Fig. S2 Specificity of the $\alpha$-MiEFF18 and pre-immune serums.

Fig. S3 Results of the yeast two-hybrid screen using MiEFF18 as a bait against the tomato root cDNA library.

Fig. S4 Minc16401 encodes a putative effector targeting the plant cell nucleus and nucleoli.

Fig. S5 Bimolecular fluorescence complementation (BiFC) experiments in N. benthamiana cells showed that S1SmD1 interact with MiEFF18, but not with MiEFF16.

Fig. S6 MiEFF18-expression in Arabidopsis transgenic lines altered M. incognita reproduction.

Fig. S7 MiEFF18 and SmD1b modulate alternative splicing in Arabidopsis roots.

Fig. S8 Effect of MiEFF 18 expression and $s m d 1 b$ mutation on AS are positively correlated.

Fig. S9 Example of alternative splicing qPCR validation for the U1-70K mRNA isoform.

Fig. S10 Venn diagrams of differentially expressed genes (DEG) in roots of the MiEFF18expressing line, the smd $1 \mathrm{~b}$ mutant and in $\mathrm{M}$. incognita-induced galls at 5 or $7 \mathrm{dpi}$.

Fig. S11 Gene ontology (GO)-term enrichment of differentially expressed genes (DEG) in the MiEFF18-expressing line and the smd $1 b$ mutant. 


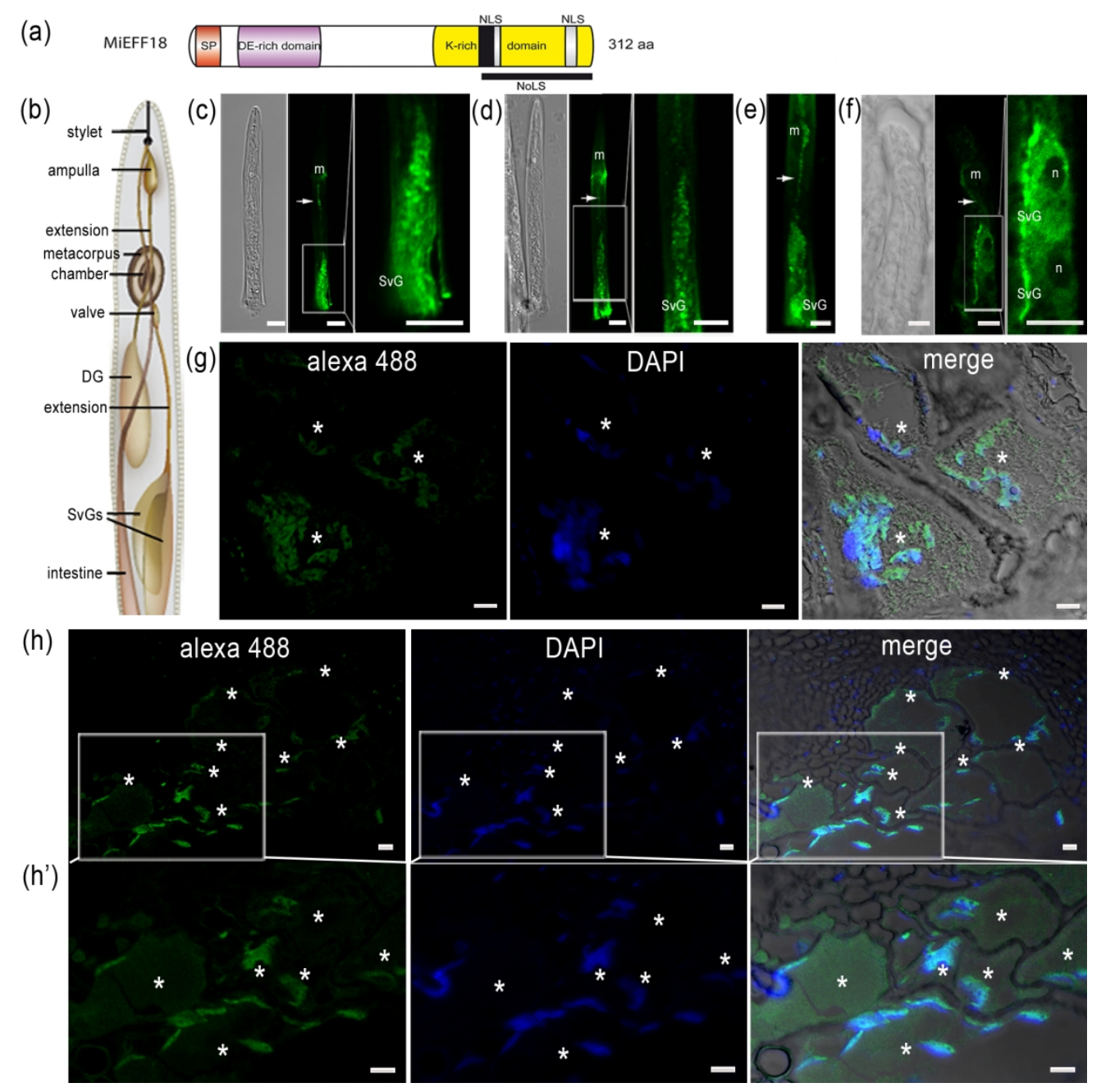

Figure 1 


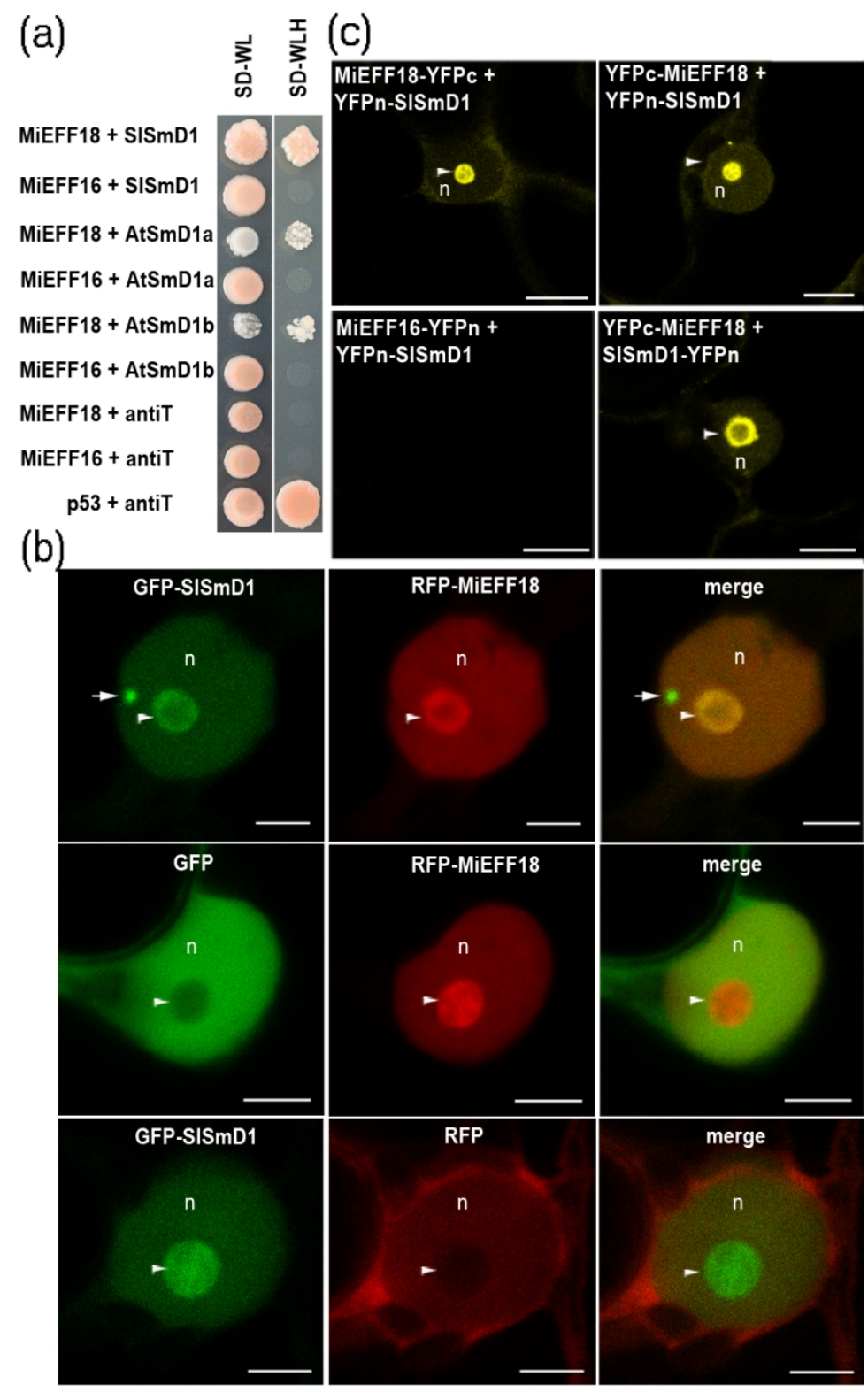

Figure 2 
(a)

\begin{tabular}{|c|c|c|c|c|c|c|c|c|c|c|}
\hline \multirow[t]{2}{*}{ (a) } & \multicolumn{2}{|c|}{ EFF18 } & \multicolumn{2}{|c|}{$s m d 1 b$} & \multicolumn{2}{|c|}{ EFF18 AND smd1b } & \multicolumn{2}{|c|}{ gall $5 \mathrm{dpi}$} & \multicolumn{2}{|c|}{ gall $7 \mathrm{dpi}$} \\
\hline & Genes & $\%$ & Genes & $\%$ & Genes & $\%$ & Genes & $\%$ & No. genes & $\%$ \\
\hline Intron retention (IR) & 76 & 34 & 234 & 51 & 36 & 34 & 80 & 19 & 96 & 22 \\
\hline Exon skipping (ES) & 18 & 8 & 37 & 8 & 9 & 8 & 12 & 3 & 24 & 5 \\
\hline Alternative 5 ' splice site (A5) & 49 & 22 & 90 & 19 & 24 & 22 & 104 & 25 & 97 & 22 \\
\hline Alternative $3^{\prime}$ splice site (A3) & 98 & 44 & 159 & 34 & 43 & 40 & 247 & 60 & 266 & 60 \\
\hline Mutually exclusive exons (MX) & 0 & 0 & 2 & 0 & 0 & 0 & 0 & 0 & 1 & 0 \\
\hline Total & 222 & 100 & 463 & 100 & 107 & 100 & 411 & 100 & 443 & 100 \\
\hline
\end{tabular}

(b)

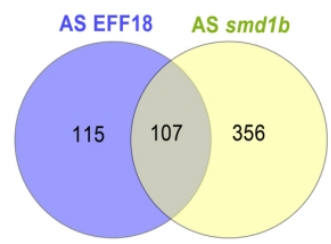

(e)
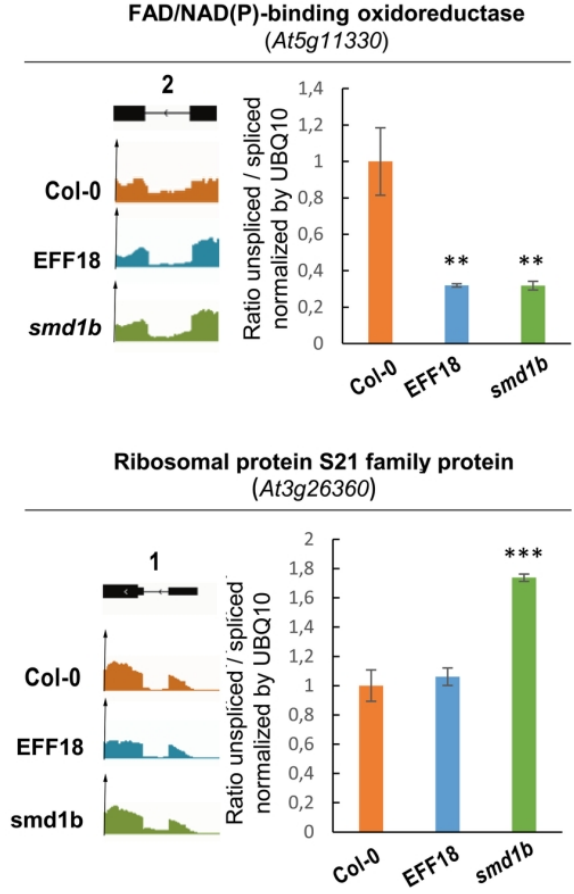

(c)

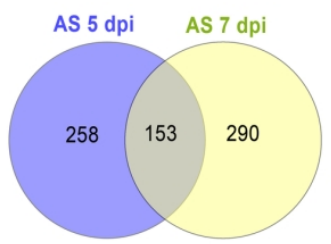

(d)

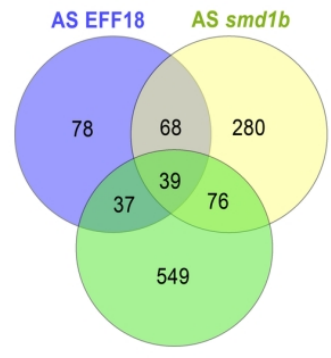

AS 5-7 dpi
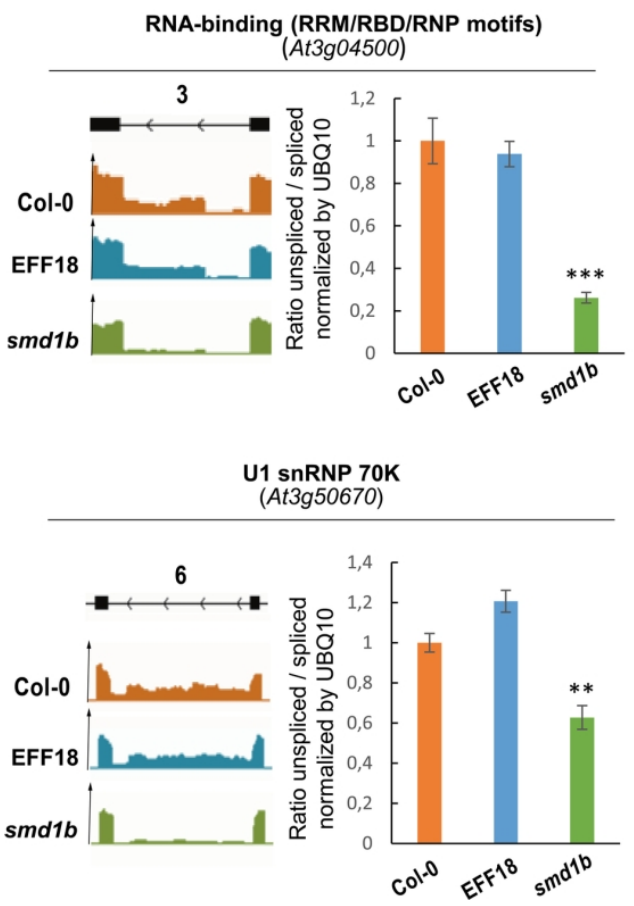

Figure 3

$160 \times 195 \mathrm{~mm}(300 \times 300$ DPI $)$ 

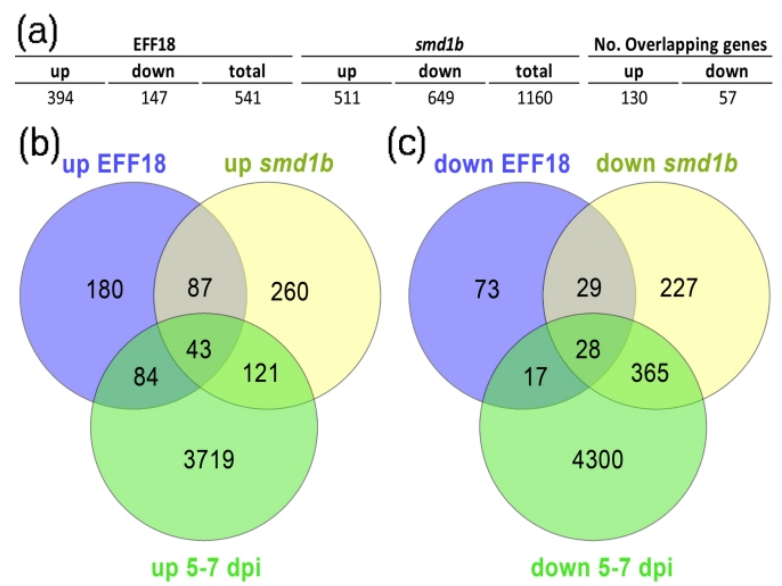

(C) down EFF18 down smd1b
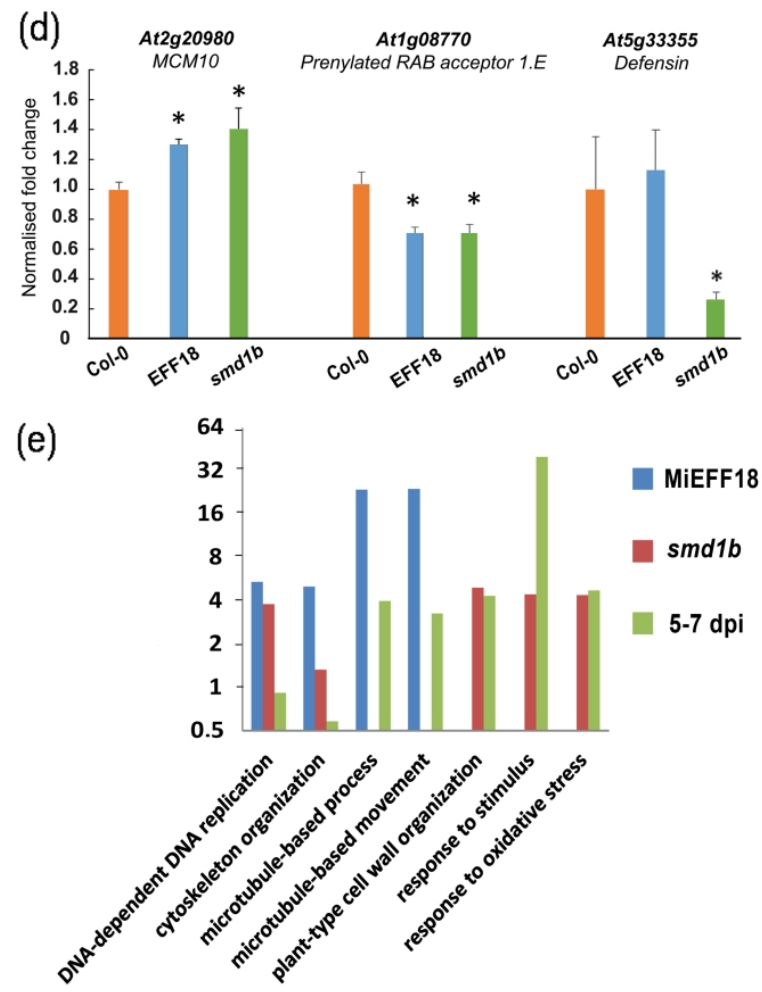

Figure 4

$80 \times 164 \mathrm{~mm}(600 \times 600 \mathrm{DPI})$ 

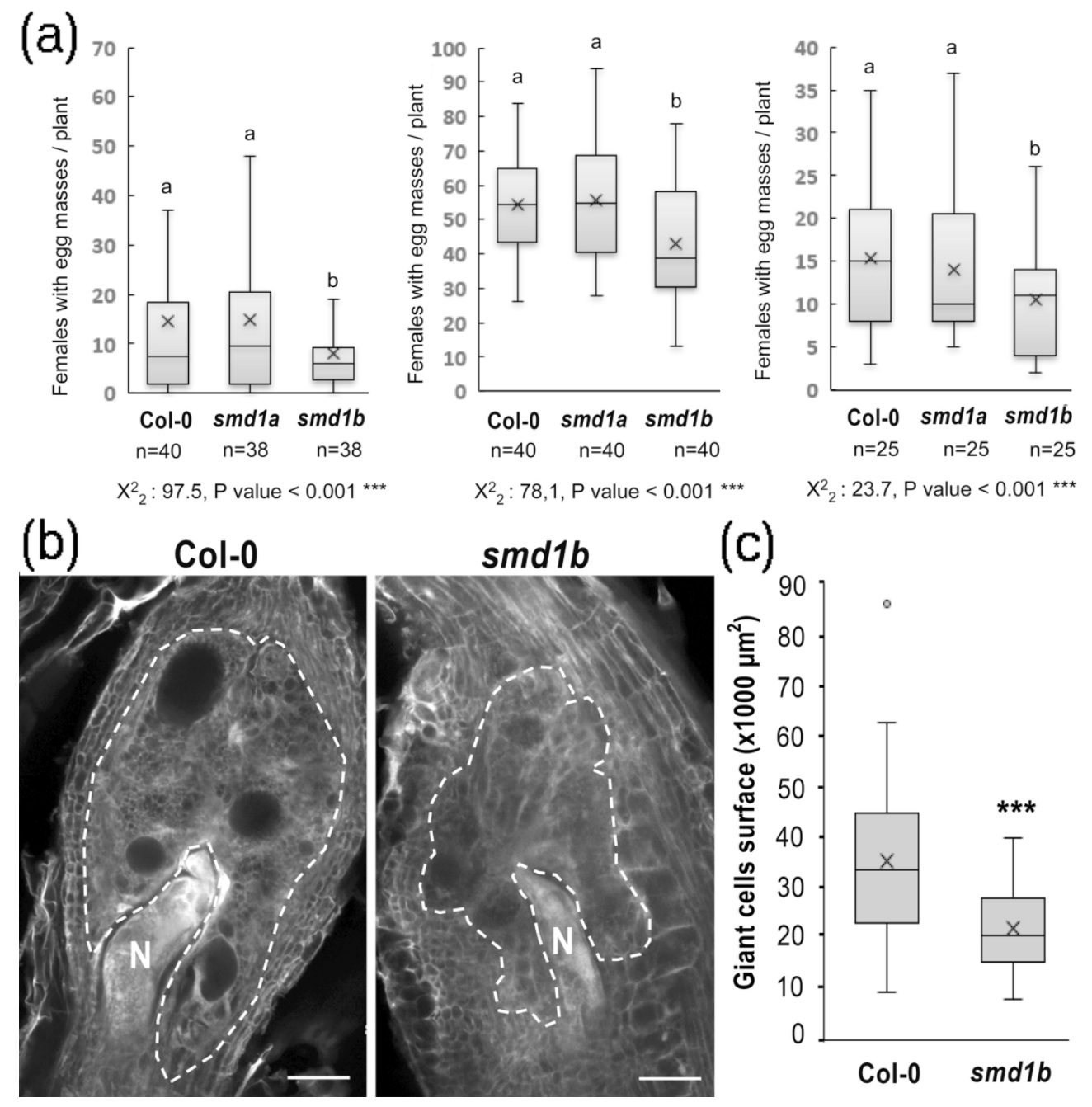

Figure 5

$80 \times 81 \mathrm{~mm}(600 \times 600 \mathrm{DPI})$ 